\title{
Attached flow structure and streamwise energy spectra in a turbulent boundary layer
}

\author{
S. Srinath, ${ }^{1}$ J. C. Vassilicos, ${ }^{1,2}$ C. Cuvier, ${ }^{1}$ J.-P. Laval, ${ }^{1}$ M. Stanislas, ${ }^{1}$ and J.-M. Foucaut ${ }^{1}$ \\ ${ }^{1}$ CNRS, ONERA, Centrale Lille, Univ. Lille, Arts et Metiers ParisTech, FRE2017, Laboratoire de Mécanique des Fluides de Lille-Kampé de \\ Fériet (LMFL), 59000 Lille, France \\ ${ }^{2}$ Department of Aeronautics, Imperial College London, London SW7 2AZ, United Kingdom
}

(Received 13 November 2017; published 14 May 2018)

\begin{abstract}
On the basis of (i) particle image velocimetry data of a turbulent boundary layer with large field of view and good spatial resolution and (ii) a mathematical relation between the energy spectrum and specifically modeled flow structures, we show that the scalings of the streamwise energy spectrum $E_{11}\left(k_{x}\right)$ in a wave-number range directly affected by the wall are determined by wall-attached eddies but are not given by the Townsend-Perry attached eddy model's prediction of these spectra, at least at the Reynolds numbers $\operatorname{Re}_{\tau}$ considered here which are between $10^{3}$ and $10^{4}$. Instead, we find $E_{11}\left(k_{x}\right) \sim k_{x}^{-1-p}$ where $p$ varies smoothly with distance to the wall from negative values in the buffer layer to positive values in the inertial layer. The exponent $p$ characterizes the turbulence levels inside wall-attached streaky structures conditional on the length of these structures. A particular consequence is that the skin friction velocity is not sufficient to scale $E_{11}\left(k_{x}\right)$ for wave numbers directly affected by the wall.
\end{abstract}

DOI: 10.1103/PhysRevE.97.053103

\section{INTRODUCTION}

In the past 40 years, the turbulence spectrum of velocity fluctuations in wall turbulence has received considerable attention as it gives valuable insight into the behavior of wall-bounded flows by indicating the distributions of energy across scales. Spectral scaling laws built on ideas initiated by Townsend [1], in particular the attached eddy hypothesis, have seen consistent development over the years (see Perry and Chong [2], Perry et al. [3], Perry \& Li [4], Marusic et al. [5], and Marusic \& Kunkel [6]). Perry and Abell [7] and Perry et al. [3] showed how Townsend's attached eddy hypothesis implies that the energy spectrum $E_{11}\left(k_{x}\right)$ of the turbulent streamwise fluctuating velocity at a distance $y$ from the wall scales as $E_{11}\left(k_{x}\right) \sim U_{\tau}^{2} k_{x}^{-1}$ in the range $1 / \delta \ll k_{x} \ll 1 / y$ where $U_{\tau}$ is the friction velocity and $\delta$ is the boundary layer thickness. Nickels et al. [8] stressed the use of overlap arguments to deduce the -1 power-law behavior. That is, a $k_{x}^{-1}$ region in the spectra would exist where the inner scaling (based on $y$ and $U_{\tau}$ ) and outer scaling (based on $\delta$ and $U_{\tau}$ ) are simultaneously valid over the same wave-number range. Nickels et al. [9] stated that it is necessary to take measurements surprisingly close to the wall to observe a $k_{x}^{-1}$ behavior and thought this was the reason why Morrison et al. [10] and McKeon and Morrison [11] did not observe any -1 region in their spectra as their measurements were not close enough to the wall. However, recent experiments by Vallikivi et al. [12] do not show an overlap region and these authors infer that the $k_{x}^{-1}$ region cannot be expected even at very high Reynolds numbers.

The present work looks at the basis for the $k_{x}^{-1}$ range in flat plate turbulent boundary layers from a new perspective. Using particle image velocimetry (PIV) and a simple model which can in principle be applied to various wall-bounded turbulent flows, we show how, in the turbulent boundary layer, a power-law spectral range exists but is not a Townsend-Perry $k_{x}^{-1}$ range and how it can be accounted for by taking only streamwise lengths and intensities of wall-attached structures into account.

This paper is organized as follows. In Secs. II and III we provide a model for the streamwise energy spectrum. The experimental setup of the flat plate boundary layer is presented in Sec. IV. Our data set is validated in Sec. V A and the method for educing the wall-attached flow structures relevant to our model is described in Sec. VB. The main results of the paper are in Secs. V C and V D followed by a discussion in Sec. VE. We conclude in Sec. VI.

\section{A SIMPLE MODEL FOR THE SPECTRAL SIGNATURE OF THE TOWNSEND-PERRY ATTACHED EDDY RANGE OF WAVE NUMBERS}

As already mentioned in the introduction, Perry and Abell [7], Perry and Chong [2], and Perry et al. [3] showed how Townsend's attached eddy hypothesis implies $E_{11}\left(k_{x}\right) \sim$ $U_{\tau}^{2} k_{x}^{-1}$ in the range $1 / \delta \ll k_{x} \ll 1 / y$. Perry et al. [3] also developed a flow structure model for this spectral range in terms of specific attached eddies of varying sizes randomly distributed in space and with a number density that is inversely proportional to size. In this paper we attempt to distill such a type of model to its bare essentials. These bare essentials are that flow structures are primarily objects with clear spatial boundaries. In Sec. V we model these boundaries with on-off functions in the expectation that the spectral signature in the attached eddy wave-number range is dominated by these sharp gradient, effectively on-off, behaviors. The concomitant expectation is that the additional superimposed velocity fluctuations fill the content of a predominantly higher frequency spectral range. In this section we show that the streamwise energy spectrum's $k_{x}^{-1}$ spectral range can be captured by simple on-off representations of elongated streaky structures of varying sizes as long as their number density has a space-filling power-law dependence on size. 
We therefore assume that the attached eddies responsible for the $k_{x}^{-1}$ spectral range have a long streaky structure footprint on the one-dimensional (1D) streamwise fluctuating velocity signals at a distance $y$ from the wall. We also assume that these streaky structures can be modeled as simple on-off functions and that it is sufficient to represent the streamwise velocity fluctuations $u(x)$ at a given height $y$ from the wall as follows:

$$
u(x)=\sum_{n, m} a_{n m} \Pi(\xi)
$$

where $\Pi(\xi)=1$ if $-1<\xi<1$ with $\xi=\frac{x-x_{n m}}{\lambda_{n}}$ and $\Pi(\xi)=0$ otherwise. The on-off function $\Pi(\xi)$ is our cartoon model of a streaky structure. Streaky structures of length $\lambda_{n}$ are centered at random positions $x_{n m}$ and their intensity is given by the coefficients $a_{n m}$. For each subscript $n$, the subscript $m$ counts the spatial positions where cartoon structures of size $\lambda_{n}$ can be centered in a given realization. The sum in (1) is over all structure lengths $\lambda_{n}$ and all their positions $x_{n m}$.

The energy spectrum of $u(x)$ is $E_{11}\left(k_{x}\right)=\frac{(2 \pi)^{2}}{L_{x}} \overline{\left|\hat{u}\left(k_{x}\right)\right|^{2}}$ where $L_{x}$ is the length of the record, $\hat{u}\left(k_{x}\right)$ is the Fourier transform of $u(x)$, and the overbar signifies an average over realizations. The Fourier transform of $\Pi\left(\frac{x-x_{n m}}{\lambda_{n}}\right)$ being $\hat{\Pi}\left(k_{x}, \lambda_{n}, x_{n m}\right)=2 i k_{x}^{-1} e^{i k_{x} x_{n m}} \sin \left(k_{x} \lambda_{n}\right)$, it follows that

$$
\hat{u}\left(k_{x}\right)=2 i k_{x}^{-1} \sum_{n m} a_{n m} e^{i k_{x} x_{n m}} \sin \left(k_{x} \lambda_{n}\right),
$$

which implies that the energy spectrum is given by

$$
\begin{aligned}
E_{11}\left(k_{x}\right) & =4 \frac{(2 \pi)^{2}}{L_{x}} k_{x}^{-2} \\
& \times \frac{\sum_{n m} a_{n m} e^{i k_{x} x_{n m}} \sin \left(k_{x} \lambda_{n}\right) \sum_{p q} a_{p q} e^{-i k_{x} x_{p q}} \sin \left(k_{x} \lambda_{p}\right)}{} .
\end{aligned}
$$

We introduce two assumptions which were also used by Perry et al. [3] in their more intricate model. The first assumption is that the positions and amplitudes of our cartoon structures are uncorrelated and that different positions are not correlated to each other either, i.e., $\overline{e^{i k_{x} x_{n m}} e^{i k_{x} x_{p q}}}=\delta_{p n} \delta_{q m}$. As a result, the expression for the energy spectrum simplifies as follows:

$$
E_{11}\left(k_{x}\right)=4 \frac{(2 \pi)^{2}}{L_{x}} k_{x}^{-2} \sum_{n m} \overline{\left(a_{n m}\right)^{2}} \sin ^{2}\left(k_{x} \lambda_{n}\right) .
$$

Let us say that there is an average number $N_{n}$ of cartoon structures of size $\lambda_{n}$ centered within an integral scale along the $x$ axis. The expression for $E_{11}\left(k_{x}\right)$ simplifies even further:

$$
E_{11}\left(k_{x}\right)=4 \frac{(2 \pi)^{2}}{L_{x}} k_{x}^{-2} \sum_{n} \overline{a_{n}^{2}} N_{n} \sin ^{2}\left(k_{x} \lambda_{n}\right),
$$

where $\overline{a_{n}^{2}} \equiv \overline{\left(a_{n m}\right)^{2}}$ is the same irrespective of position $x_{n m}$.

We now consider a continuum of different structure sizes $\lambda$ rather than discrete length scales $\lambda_{n}$ and the previous expression for $E_{11}\left(k_{x}\right)$ must therefore be replaced by

$$
E_{11}\left(k_{x}\right)=4 \frac{(2 \pi)^{2}}{L_{x}} k_{x}^{-2} \int d \lambda \overline{a^{2}}(\lambda) N(\lambda) \sin ^{2}\left(k_{x} \lambda\right)
$$

in terms of easily understandable notation. At this point we introduce a generalized form of the second assumption which was also used by Perry et al. [3]: We assume a power-law form for $N(\lambda)$ in the range $\lambda_{i}<\lambda<\lambda_{o}$ where $\lambda_{i} \sim y$ and $\lambda_{o} \sim \delta$, and $N(\lambda)=0$ outside this range for simplicity. This power-law form is $N(\lambda)=\left(-N_{M}+N_{o}(\lambda / \delta)^{-1-D}\right)$ where $N_{M}$ and $N_{o}$ are positive dimensionless numbers which increase proportionally to $L_{x}$ so as to keep number densities constant. The number $N_{M}$ is introduced to allow for the possibility of an upper bound on streaky structure size given by $N\left(\lambda_{o}\right)=0$, i.e., $N_{M}=N_{o}\left(\lambda_{o} / \delta\right)^{-1-D}$ which should be small given that LSM and VLSM streaky structures have been observed with lengths greater than $\delta$ (see Smits et al. [13]).

Vassilicos and Hunt [14] proved that, if $0 \leqslant D \leqslant 1$, then the set of points defining the edges of the on-off functions $\Pi(\xi)$ is fractal and $D$ is effectively the fractal dimension of this set of points. The case where this fractal dimension is $D=1$ is the case where these points are space filling. The population density assumption of Perry et al. [3] corresponds to $D=1$ which is also the choice we make in this work. We now show that this choice can lead to $E_{11}\left(k_{x}\right) \sim k_{x}^{-1}$ in the range $1 / \lambda_{o} \ll k_{x} \ll 1 / \lambda_{i}$.

We calculate the energy spectrum by carrying out the integral in (6). This requires a model for $\overline{a^{2}}(\lambda)$ which, in this section, we chose to be as simple as possible and therefore independent of $\lambda$ in the relevant range, i.e., $\overline{a^{2}}(\lambda)=A^{2} / \delta$ for $\lambda_{i}<\lambda<\lambda_{o}$ where $A^{2}$ is a constant. Using our models for $N(\lambda)$ and $\overline{a^{2}}(\lambda)$ and the change of variables $\lambda k_{x}=l$, (6) becomes

$$
E_{11}\left(k_{x}\right)=A^{2} \delta\left(C_{o}\left(k_{x} \delta\right)^{-2+D}-C_{M}\left(k_{x} \delta\right)^{-2}\right),
$$

where

$$
C_{o}=4(2 \pi)^{2} N_{o} \frac{\delta}{L_{x}} \int_{\lambda_{i} k_{x}}^{\lambda_{o} k_{x}} d l \sin ^{2}(l) l^{-1-D}
$$

and

$$
C_{M}=4(2 \pi)^{2} N_{M} \frac{\delta}{L_{x}}\left(k_{x} \delta\right)^{-1} \int_{\lambda_{i} k_{x}}^{\lambda_{o} k_{x}} d l \sin ^{2}(l),
$$

which is bounded from above by $\frac{N_{M}}{L_{x}} \frac{\lambda_{o}-\lambda_{i}}{\delta}$. In the attached eddy range $1 / \lambda_{o} \ll k_{x} \ll 1 / \lambda_{i}, C_{o} \approx 4(2 \pi)^{2} \frac{N_{o}}{L_{x}} \int_{0}^{\infty} d l \sin ^{2}(l) l^{-1-D}$ which means that $C_{o}$ is approximately independent of $k_{x}$ in this range.

Substituting the value $D=1$ in Eq. (7), we get $E_{11}\left(k_{x}\right)=$ $A^{2}\left(C_{o} k_{x}^{-1}-C_{M} \delta^{-1} k_{x}^{-2}\right)$ which is well approximated by

$$
E_{11}\left(k_{x}\right) \approx C_{o} A^{2} k_{x}^{-1}
$$

for wave numbers $k_{x} \delta \gg C_{M} / C_{o}$ (i.e., $C_{o} k_{x}^{-1} \gg C_{m} \delta^{-1} k_{x}^{-2}$ ). Note that $C_{M} / C_{o}$ is much smaller than 1 because $N_{M}$ is much smaller than $N_{o}$ and that (8) is valid in the range $1 / \lambda_{o} \ll k_{x} \ll$ $1 / \lambda_{i}$ where $\lambda_{o}$ scales with but is much larger than $\delta$. For a good correspondence with the scalings of the Townsend-Perry attached eddy model one needs to take $\lambda_{i} \sim y$ and $A^{2} \sim U_{\tau}^{2}$. 


\section{A STRAIGHTFORWARD GENERALIZATION}

It is worth generalizing the previous section's model by assuming that $\overline{a^{2}}(\lambda)$ is not constant but varies with $\lambda$ in the range $\lambda_{i}<\lambda<\lambda_{o}$, for example, as $\overline{a^{2}}(\lambda)=\left(A^{2} / \delta\right)(\lambda / \delta)^{p}$ where $p$ is a real number with bounds which we determine below. The arguments of the previous section can be reproduced until Eq. (6) which now becomes

$$
E_{11}\left(k_{x}\right)=A^{2} \delta\left[c_{o}\left(k_{x} \delta\right)^{-2+D-p}-c_{M}\left(k_{x} \delta\right)^{-2}\right]
$$

where

$$
c_{o}=4(2 \pi)^{2} N_{o} \frac{\delta}{L_{x}} \int_{\lambda_{i} k_{x}}^{\lambda_{o} k_{x}} d l \sin ^{2}(l) l^{-1-D+p}
$$

and

$$
c_{M}=4(2 \pi)^{2} N_{M} \frac{\delta}{L_{x}}\left(k_{x} \delta\right)^{-1-p} \int_{\lambda_{i} k_{x}}^{\lambda_{o} k_{x}} d l l^{+p} \sin ^{2}(l),
$$

which is bounded from above by $\frac{N_{m}}{(1+p) L_{x}}\left[\left(\frac{\lambda_{o}}{\delta}\right)^{1+p}-\left(\frac{\lambda_{i}}{\delta}\right)^{1+p}\right]$. In the attached eddy range $1 / \lambda_{o} \ll k_{x} \ll 1 / \lambda_{i}, \quad c_{o} \approx$ $4(2 \pi)^{2} \frac{N_{o}}{L_{x}} \int_{0}^{\infty} d l \sin ^{2}(l) l^{-1-D+p}$ which means that $c_{o}$ is approximately independent of $k_{x}$ in this range if $0<D-p<2$.

Substituting the value $D=1$ in (9), we obtain the following leading order approximation in the parameter range $-1<p<1$ :

$$
E_{11}\left(k_{x}\right) \approx c_{0} A^{2} \delta\left(k_{x} \delta\right)^{q},
$$

where

$$
p+q=-1
$$

for wave numbers $k_{x} \delta \gg\left(c_{M} / c_{o}\right)^{\frac{1}{1-p}}$. Note that $c_{M} / c_{o}$ is much smaller than 1 if $p$ is not too close to 1 because $N_{M}$ is much smaller than $N_{o}$.

The spectral shape (10) is potentially significantly different from what the classical Townsend-Perry attached eddy model predicts. We emphasize that in this and the previous sections we have developed a simple model based on on-off functions representing long streaky structures which returns a wavenumber dependency of $E_{11}\left(k_{x}\right)$ which is either identical to the Townsend-Perry spectral shape if $p=0$, or different but in some ways comparable if $p \neq 0$. In the remainder of this paper we present experimental evidence in support of $D=1$, and (10) and (11) rather than (8), with $p$ as a function of $y^{+}$.

\section{EXPERIMENTAL SETUP}

An experiment was performed in the boundary layer wind tunnel at the Lille Fluid Mechanics Laboratory (LMFL) having a test section 2-m wide, 1-m high, and 20.6-m long. The tests were conducted at two free stream velocities of 3 $\mathrm{m} / \mathrm{s}$ and $10 \mathrm{~m} / \mathrm{s}$ corresponding to Reynolds numbers $\operatorname{Re}_{\theta}=$ $8100\left(\operatorname{Re}_{\tau}=2700\right)$ and $\operatorname{Re}_{\theta}=20600\left(\operatorname{Re}_{\tau}=7200\right)$, respectively. To capture the large streamwise wall-normal field, four 12-bits Hamamatsu cameras having a resolution of $2048 \times 2048$ pixels were installed in a series to observe a region between $19.26 \mathrm{~m}$ and $20.42 \mathrm{~m}$ from the inlet which is $1.16-\mathrm{m}$ long $\left(\approx 3.36 \delta\right.$ and $3.85 \delta$, for $\operatorname{Re}_{\theta}=8100$ and 20600 , respectively) and $0.3-\mathrm{m}$ high $\left(\approx 0.86 \delta\right.$ and $1 \delta$ for $\operatorname{Re}_{\theta}=8100$ and 20600 , respectively).
Nikon lenses of 50-mm focal length were set on the cameras and the magnification obtained was 0.05 . The f stop of 5.6 leads to a diffraction spot size of $7.7 \mu \mathrm{m}$ which is slightly higher than 1 pixel and a depth of field of $18 \mathrm{~mm}$. The uncertainties were estimated in the merged regions of two PIV systems (see Kostas et al. [15]) to be slightly less than $1 \%$ of free stream velocity which corresponds to about 0.1 pixel as obtained from the spectral analysis. The software HIRIS was used to acquire the images of the four cameras simultaneously. A total of 22500 and 29500 samples were recorded at the highest and lowest Reynolds numbers, respectively. The flow was seeded with $1 \mu \mathrm{m}$ poly-ethylene glycol and illuminated by a double pulsed NdYAG laser at $400 \mathrm{~mJ} /$ pulse and the laser sheet thickness was of the order of $1 \mathrm{~mm}$ within the full field of view.

The modified version by LML of the MATPIV toolbox, was used under MATLAB to process the acquired images from the 2D2C PIV. The mean background images were mapped using a basic pinhole model and the laser reflection (i.e., wall position) was fitted with a simple line. A mesh was then built above this line in the mapped images (spacing of about 1.5 by $1.5 \mathrm{~mm}$ corresponding to about 10 pixels) and projected on each camera with the pinhole models. The PIV analysis was then done with these projected grids. Background division [16] was used to limit the effect of the laser reflection and the camera noise. The cross-correlation PIV analysis (Willert and Gharib [17] and Soria [18]) was done with four passes (one $64 \times 64$, two $32 \times 32$, and a final one $28 \times 28$ ). Before the final pass, image deformation (Scarano [19] and Lecordier and Trinite [20]) was used with a cubic B-spline interpolation of the gray level and bilinear interpolation for the displacement to improve the quality of the final result. The data validation is based on the median method (Westerweel and Scarano [21]). The final grid had 766 points along the wall and 199 points in the wall-normal direction with a grid spacing of $1.5 \mathrm{~mm}$ corresponding to 11 wall units and 35 wall units for the test cases at $\operatorname{Re}_{\theta}=8100$ and $\operatorname{Re}_{\theta}=20600$, respectively.

\section{RESULTS AND DISCUSSION}

\section{A. Validation of experimental data}

Figure 1 shows profiles of the mean streamwise velocity $U$ and the rms streamwise fluctuating velocity $u^{\prime}$ obtained from PIV at $\operatorname{Re}_{\theta}=8100$ and $\operatorname{Re}_{\theta}=20600$ and compared with the hot-wire anemometry results of Carlier and Stanislas [22]. The mean velocity profiles are in good agreement with the hot-wire data and are well resolved from $y^{+} \approx 30$ and $y^{+} \approx 90$ upwards for $\operatorname{Re}_{\theta}=8100$ and 20600 , respectively. Comparisons of the profiles of $u^{\prime+}\left(u^{\prime}\right.$ scaled with inner variables) show a fairly good match with the hot-wire data. A plateau of $u^{\prime+}$ is present in the range $100<y^{+}<300$ for our higher Reynolds number case. Close to the wall, the $u^{\prime+}$ values obtained from our PIV are slightly underestimated, in particular for $\operatorname{Re}_{\theta}=20600$, demonstrating some filtering of the PIV at this resolution (Foucaut et al. [23]). To compute from PIV the energy spectra used in this paper, we used the method of Foucaut et al. [23]. As seen in Fig. 2 for the particular case of wall distance $y^{+}=200$ at $\operatorname{Re}_{\theta}=20600$, the agreement between the spatial spectrum from the PIV and the temporal spectrum from the hot-wire anemometry of Carlier and Stanislas [22] is good up 

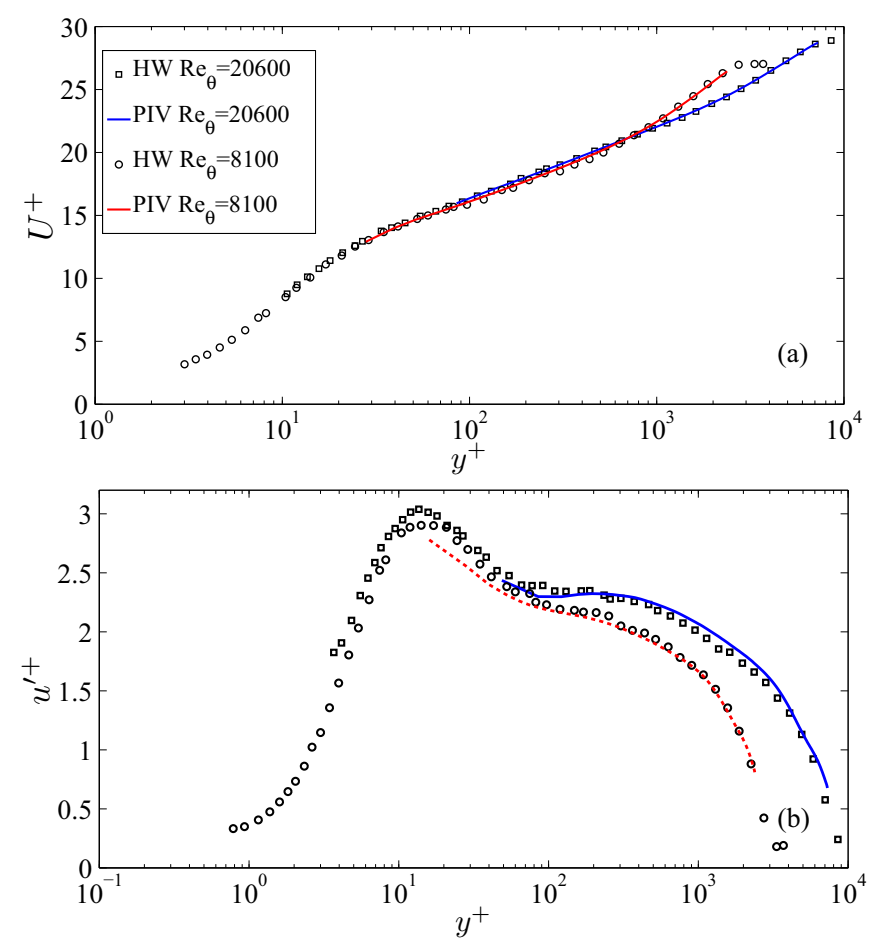

FIG. 1. Mean streamwise velocity profiles (top) and rms streamwise fluctuating velocity profiles (bottom) at $\operatorname{Re}_{\theta}=8100\left(U_{\infty}=\right.$ $3 \mathrm{~m} / \mathrm{s})$ and $\operatorname{Re}_{\theta}=20600\left(U_{\infty}=10 \mathrm{~m} / \mathrm{s}\right)$ obtained with PIV and compared with the hot wire anemometry results of Carlier and Stanislas [22].

to wave numbers $k_{x} \approx 700$ (attenuation of $3 \mathrm{db}$ ) corresponding to length scales of $9 \mathrm{~mm}$.

\section{B. Structure detection}

In Secs. II and III we developed a spectral model of the streamwise turbulence fluctuating velocity based on the concept of elongated streaky structures which are part of attached eddies and can be modeled as simple on-off functions. In this and the next subsections we use our PIV data to

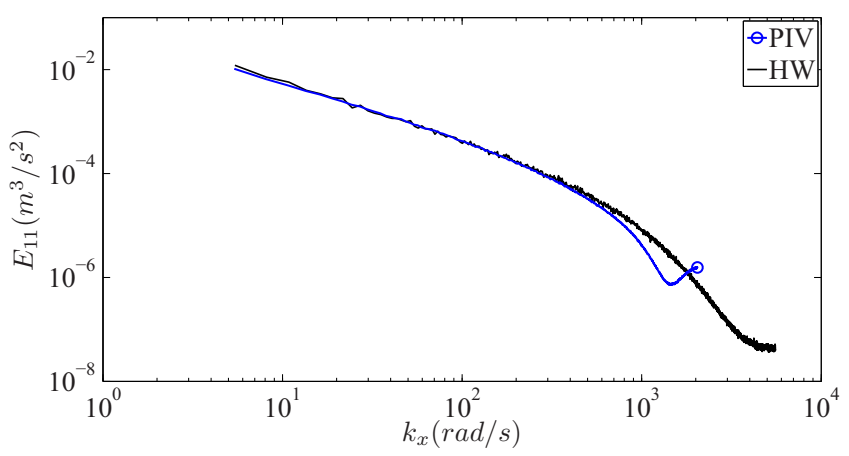

FIG. 2. Comparison of the streamwise energy spectra obtained from PIV and hot-wire anemometry at $y^{+}=200$ for $\operatorname{Re}_{\theta}=20600$. $E_{11}\left(k_{x}\right)$ is in $\mathrm{m}^{3} / \mathrm{s}^{2}$ and $k_{x}$ is in $\mathrm{rad} / \mathrm{m}$. The hot-wire anemometry was made by Carlier and Stanislas [22] at $19.6 \mathrm{~m}$ from the wind tunnel inlet in the same wind tunnel.
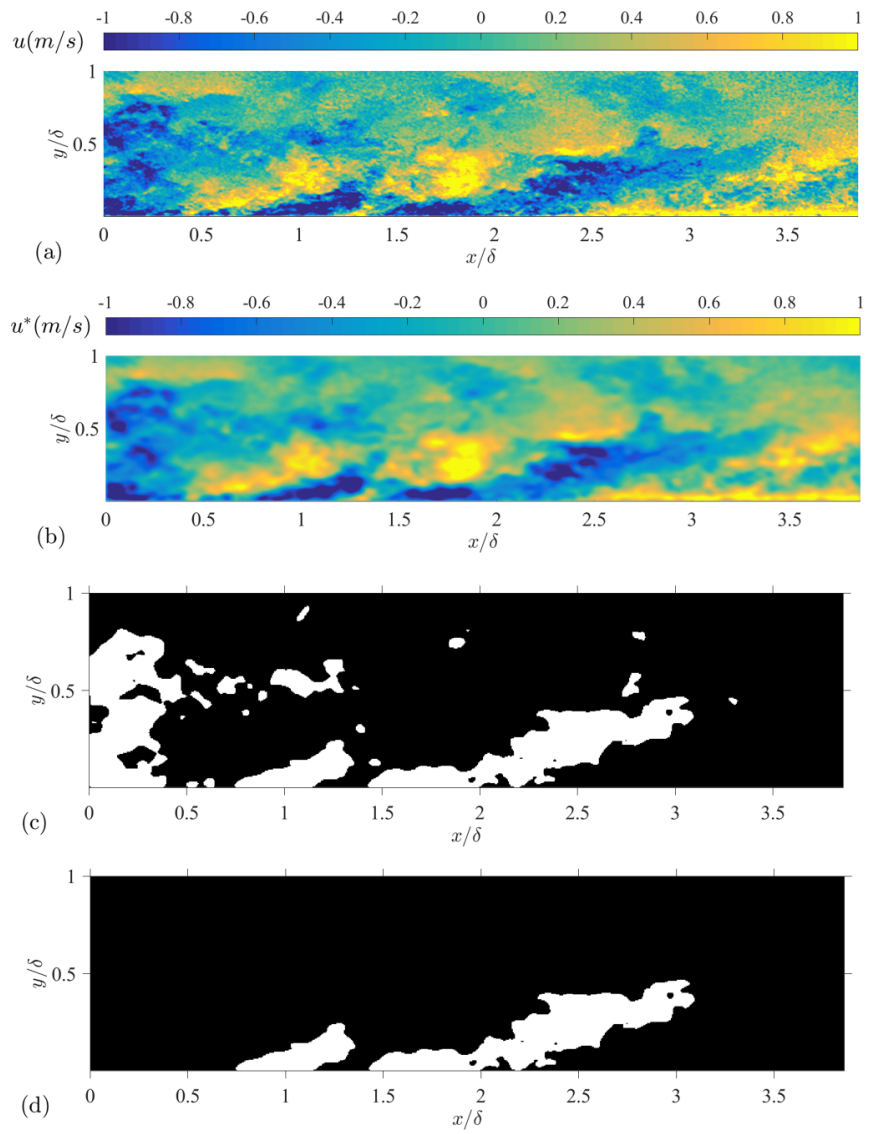

FIG. 3. Wall-attached elongated streaky structure eduction method applied on a sample instantaneous streamwise velocity field at $\operatorname{Re}_{\theta}=20600$. (a) Raw instantaneous streamwise fluctuating velocity component field. (b) The same field after application of a Gaussian filter. (c) Binary image obtained after thresholding negative streamwise fluctuating momentum regions. (d) Final image obtained after cleaning as described in Sec. VB.

test this concept and assess its potential as an hypothesis for understanding near-wall streamwise energy spectra.

Figure 3(a) shows a sample field of instantaneous streamwise fluctuating velocity components $u$. The existence of welldefined elongated and tilted wall-attached regions of relatively high (positive or negative) $u$ values is clear. It is these regions that we need to target in relation to the elongated streaky structures of our model.

The raw instantaneous streamwise velocity fields are affected by noise so that single structures in Fig. 3(a) appear split in many little parts. To smooth out these structures without modifying their shape and statistics we used a two-dimensional Gaussian filter. This filtering operation was found to be sufficient to capture and connect the structures while retaining their overall shape. The standard deviation of the Gaussian filter was three pixels which corresponds to approximately $0.015 \delta$ for both Reynolds numbers, i.e., 105 wall units for $\operatorname{Re}_{\theta}=20600$ and 33 wall units for $\operatorname{Re}_{\theta}=8100$. The result of this operation in Fig. 3(a) leads to Fig. 3(b).

To educe on-off functions such as the ones required by our model we apply a threshold $u_{\text {th }}$ on the Gaussian-filtered $u^{*}$ to obtain binary images which distinguish between $u^{*}<u_{\mathrm{th}}$ and 
$u^{*}>u_{\mathrm{th}}$. Effects of the threshold on the statistics of educed structures were investigated in the range $0.1 u_{300^{+}}^{\prime}<\left|u_{\mathrm{th}}\right|<$ $u_{300^{+}}^{\prime}$ where $u_{300^{+}}^{\prime}$ is $u^{\prime}$ at $y^{+}=300$.

A threshold $u_{\text {th }}$ equal to $-0.4 u_{300^{+}}^{\prime}$ was finally chosen to detect low momentum structures in the present study as it corresponds to the value that leads to least threshold dependency of our statistics for a negative $u_{\mathrm{th}}$ (for example, $u_{\mathrm{th}}$ equal to $-0.2 u_{300^{+}}^{\prime}$ or $-0.6 u_{300^{+}}^{\prime}$ return results with no significant difference; see Appendix). This thresholding operation leads to Fig. 3(c) when applied to Fig. 3(b). The white structures in Fig. 3(c) correspond to $u^{*}<u_{\text {th }}$.

One more step is required before comparing with our model. White structures which cut through the vertical borders of the figure are discarded because their streamwise extent is unknown; white structures which are not attached to the bottom wall (at $y=0$ but in fact as close to $y=0$ as allowed by our PIV data, i.e., $y^{+}=16$ and $y^{+}=48$ for the lower and the higher Reynolds number cases, respectively) are also discarded because we are concerned with wall-attached structures. With this extra step, Fig. 3(c) gives rise to Fig. 3(d).

All the steps leading from raw fluctuating streamwise velocity fields to the binary fields which we use in our statistical analysis are depicted in Fig. 3. The current study's effort is concentrated on wall-attached elongated structures of negative streamwise fluctuating velocity as in Fig. 3(d), but the analysis can be repeated equally well on structures of positive streamwise fluctuating velocity with results that are similar though slightly less sharp with regard to (10) and (11) (see Appendix). The general behaviors of positive and negative fluctuating streamwise velocity structures are similar, the negative velocity structures being slightly longer in agreement with Dennis and Nickels [24].

\section{Lengths of wall-attached streamwise velocity structures}

We now need to obtain statistics of wall-attached elongated streaky structures represented as on-off functions in our model and as binary structures in the final stages of our structure eduction method. We first label the connected components of the binary images using image processing tools. Then we compute the streamwise length $\lambda$ of each labeled structure at a distance $y$ from the wall, i.e., the difference between the smallest and the largest values of streamwise coordinate $x$ in this labeled structure at height $y$. Finally we compute the average value $\alpha$ of the streamwise fluctuating velocity component $u$ inside this labeled structure at height $y$. Thus we obtain a pair $(\lambda, \alpha)$ for each labeled structure at each height $y$ considered. This procedure is illustrated in Fig. 4 where the streamwise extent $\lambda$ and the corresponding amplitude $\alpha$ of two labeled structures at wall distance $y / \delta=0.03$ is shown. A total of 14493 and 19576 wall-attached binary structures were detected at $\operatorname{Re}_{\theta}=20600$ and $\operatorname{Re}_{\theta}=8100$, respectively. (As mentioned in Sec. IV, 22500 and 29500 samples were recorded at the highest and lowest of our two values of $\operatorname{Re}_{\theta}$, respectively, and the ratios $14930: 22500$ and $19576: 29500$ are about the same.)

The model in Secs. II and III assumes that the number of wall-attached elongated streaky structures of size $\lambda$ has a decreasing power-law dependence on $\lambda$ in a certain range of $\lambda$ values. Following Perry et al. [3], we expect the spatial

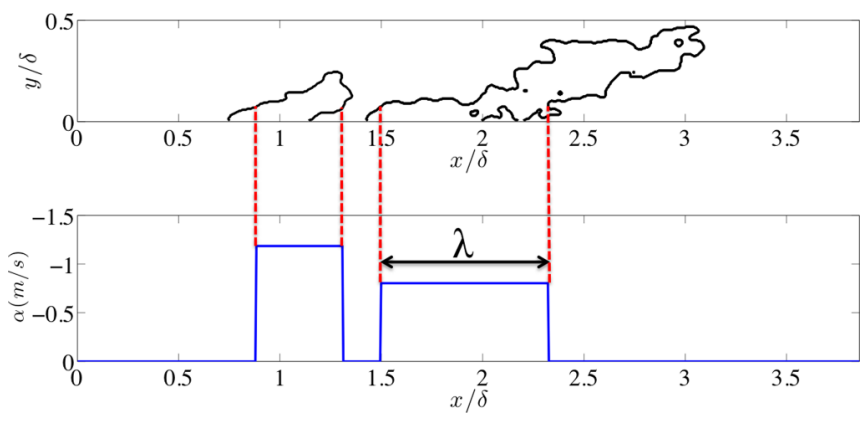

FIG. 4. Figure 3(d) reproduced in the top plot, with, in the bottom plot, the average streamwise fluctuating velocity $\alpha$ and the streamwise length $\lambda$ of the detected wall-attached structures at $y / \delta=0.03$.

distribution of such structures to be space filling, which implies (see Vassilicos and Hunt [14]) that the exponent of this power law should be -2 . Figures 5 and 6 show the probability distribution function (PDF) of lengths $\lambda$ at various wall distances. The most probable length $\lambda$ lies between $0.3 \delta$ and $0.5 \delta$ and lengths $\lambda$ longer than $3.5 \delta$ occur very rarely.

We tested for finite size effects of the field of view by computing the PDF on smaller domains, namely $3.5 \delta$ and $3 \delta$ long in the streamwise direction but the same in the wall normal direction. As shown in Fig. 5 there is no significant difference caused by the three fields of view except that the smallest field returns a slightly more noisy PDF. Indeed, a reduced field of view leads to a smaller number of detected wall-attached elongated binary structures and therefore to reduced statistical convergence.
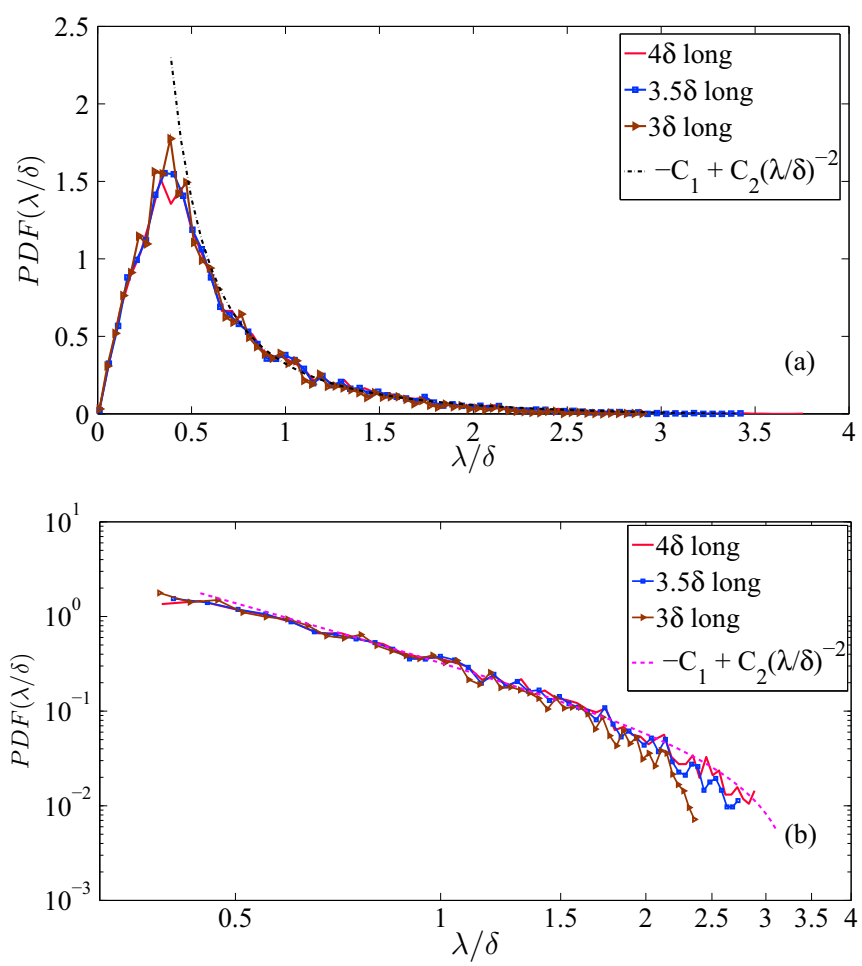

FIG. 5. PDFs of streamwise lengths $\lambda$ (see Fig. 4) for varying domain lengths at wall distance $y^{+}=195$ for $\operatorname{Re}_{\theta}=20600$. Lin-lin plot (top) and premultiplied log-log plot (bottom). 

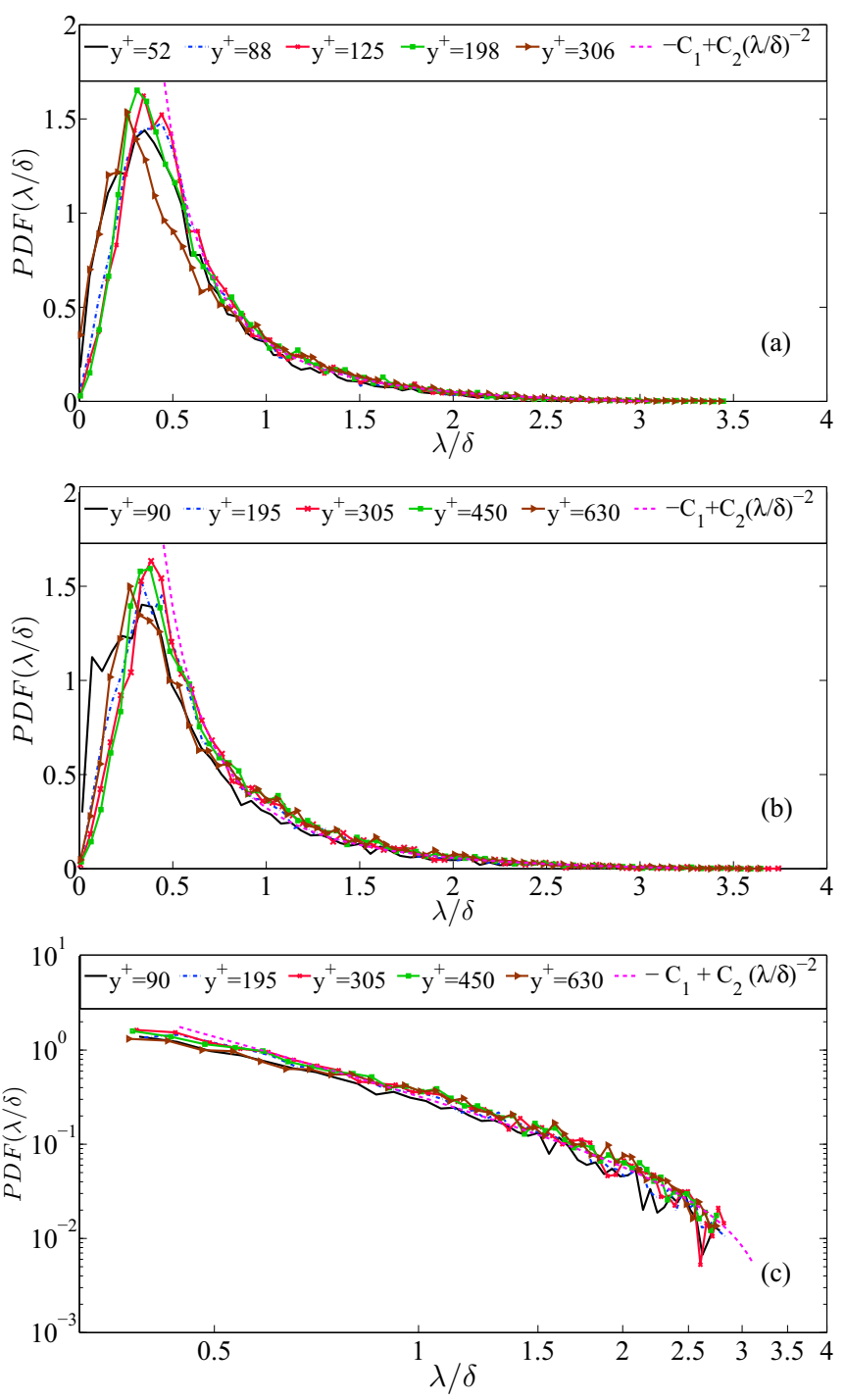

FIG. 6. PDFs of streamwise lengths $\lambda$ of wall-attached structures (see Fig. 4) at selected wall distances for $\operatorname{Re}_{\theta}=8100$ (top) and $\operatorname{Re}_{\theta}=$ 20600 (middle). The fits shown in these top and middle plots are for $y^{+}=198$ at $\operatorname{Re}_{\theta}=8100$ and $y^{+}=195$ at $\operatorname{Re}_{\theta}=20600$. The bottom plot is a log-log reproduction of the middle plot's data.

Figures 5 and 6 show a power-law dependence on $\lambda$ between about $0.5 \delta$ and $2 \delta$ with power-law exponent -2 , i.e., $D=1$, in all cases. Given the form of $N(\lambda)$ hypothesized in Secs. II and III, we fit the PDF of $\lambda / \delta$ with a functional form $-C_{1}+C_{2}(\lambda / \delta)^{-2}$ (where $C_{1} / N_{M}=C_{2} / N_{o}$ ). The fit is shown in Figs. 5 and 6 and is effectively the same for both Reynolds numbers and all values of $y^{+}$in the mean flow's approximate $\log$ region. The constants $C_{1}$ and $C_{2}$ are reported in Table I. They are indeed fairly constant over the range of wall distances and for both Reynolds numbers. Identical results are obtained for wall-attached structures with positive streamwise fluctuating velocity except that $C_{1} \approx 0.02$ for both Reynolds numbers and $C_{2} \approx 0.29$ for $\operatorname{Re}_{\theta}=8100$ (see Table III in the appendix). It is worth noting that the lower bound of the range where the PDF of $\lambda / \delta$ is well approximated by $-C_{1}+C_{2}(\lambda / \delta)^{-2}$ seems to increase slightly with increasing $y^{+}$.
TABLE I. Values of the constants $C_{1}$ and $C_{2}$ in the form $-C_{1}+$ $C_{2}(\lambda / \delta)^{-2}$ of the PDF of $\lambda / \delta$. In the $\operatorname{Re}_{\theta}=20600$ case, the fit is over a range of $\lambda / \delta$ bounded from above by 3.8 and from below by 0.49 $\left(y^{+}=90\right), 0.55\left(y^{+}=195\right), 0.54\left(y^{+}=305\right), 0.58\left(y^{+}=450\right)$, and $0.78\left(y^{+}=630\right)$. In the $\operatorname{Re}_{\theta}=8100$ case, the fit is over a range of $\lambda / \delta$ bounded from above by 3.4 and from below by $0.54\left(y^{+}=52\right), 0.53$ $\left(y^{+}=88\right), 0.53\left(y^{+}=125\right), 0.56\left(y^{+}=198\right)$, and $0.65\left(y^{+}=306\right)$.

\begin{tabular}{lcccccccccc}
\hline \hline $\operatorname{Re}_{\theta}$ & \multicolumn{1}{c}{20600} & \multicolumn{1}{c}{8100} \\
\hline$y^{+}$ & 90 & 195 & 305 & 450 & 630 & 52 & 88 & 125 & 198 & 306 \\
$C_{1}$ & 0.03 & 0.03 & 0.03 & 0.03 & 0.03 & 0.04 & 0.04 & 0.04 & 0.03 & 0.03 \\
$C_{2}$ & 0.32 & 0.35 & 0.35 & 0.37 & 0.37 & 0.32 & 0.35 & 0.35 & 0.35 & 0.33 \\
\hline \hline
\end{tabular}

\section{Energy spectra}

Figure 7 shows log-log plots of premultiplied energy spectra of streamwise fluctuating velocities $u(x)$ which have been obtained from our PIV data at various normalized wall distances $y^{+}$for both Reynolds numbers. These plots might suggest that $E_{11}\left(k_{x}\right) \sim U_{\tau}^{2} k_{x}^{-1}$ in a range of wave numbers $2 \pi /(4 \delta) \lesssim k_{x} \lesssim$ $0.63 / y$ for $y^{+}$larger than about 88 and smaller than the value of $y^{+}$where this range of wave numbers no longer exists. The apparent $k_{x}^{-1}$ wave-number range is close to a decade long at $y^{+}=90$ for $\operatorname{Re}_{\theta}=20600$ and shorter for higher wall normal distances and for the lower Reynolds number $\left(\operatorname{Re}_{\theta}=8100\right)$. One would be justified to conclude that this is indeed experimental support for the Townsend-Perry $k_{x}^{-1}$ spectrum if the only available theoretical glasses through which to look at these spectral plots were those of the Townsend-Perry attached eddy model. However, the situation is subtler and, in effect, quite different.
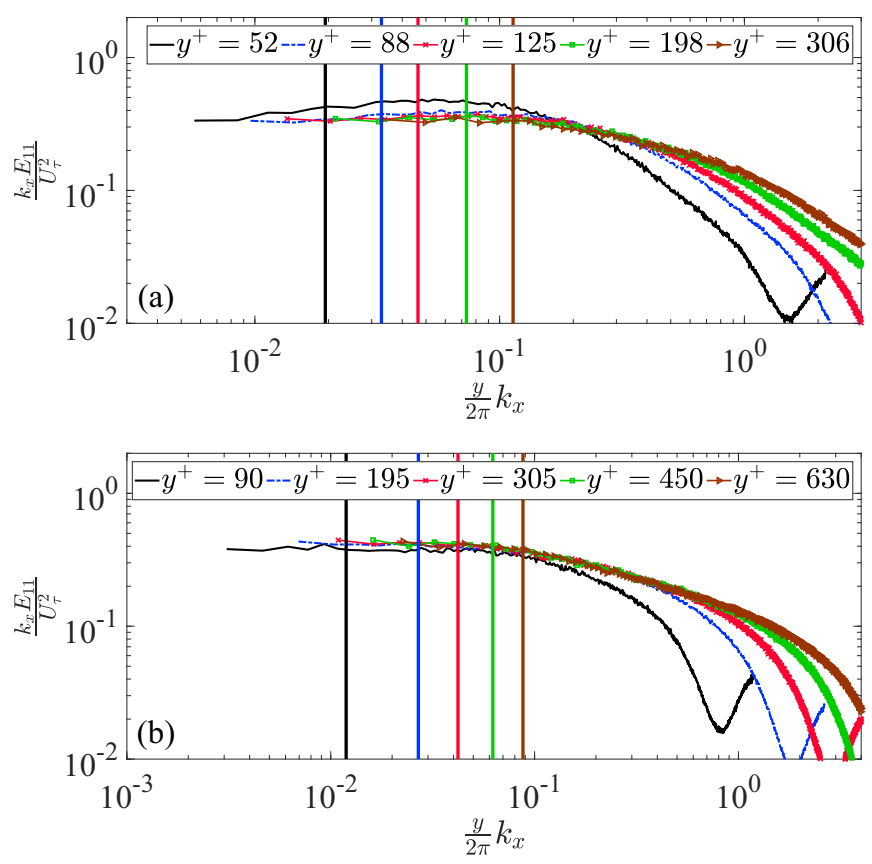

FIG. 7. Log-log plots of premultiplied streamwise energy spectra at selected wall distances for $\operatorname{Re}_{\theta}=8100$ above and $\operatorname{Re}_{\theta}=20600$ below. Vertical lines show $\frac{y}{2 \pi} k_{x}=(y / 2 \pi)(2 \pi / \delta)=\frac{y}{\delta}$ (corresponding to $k_{x}=2 \pi / \delta$ ) with the same color code as the legend of the figure. 

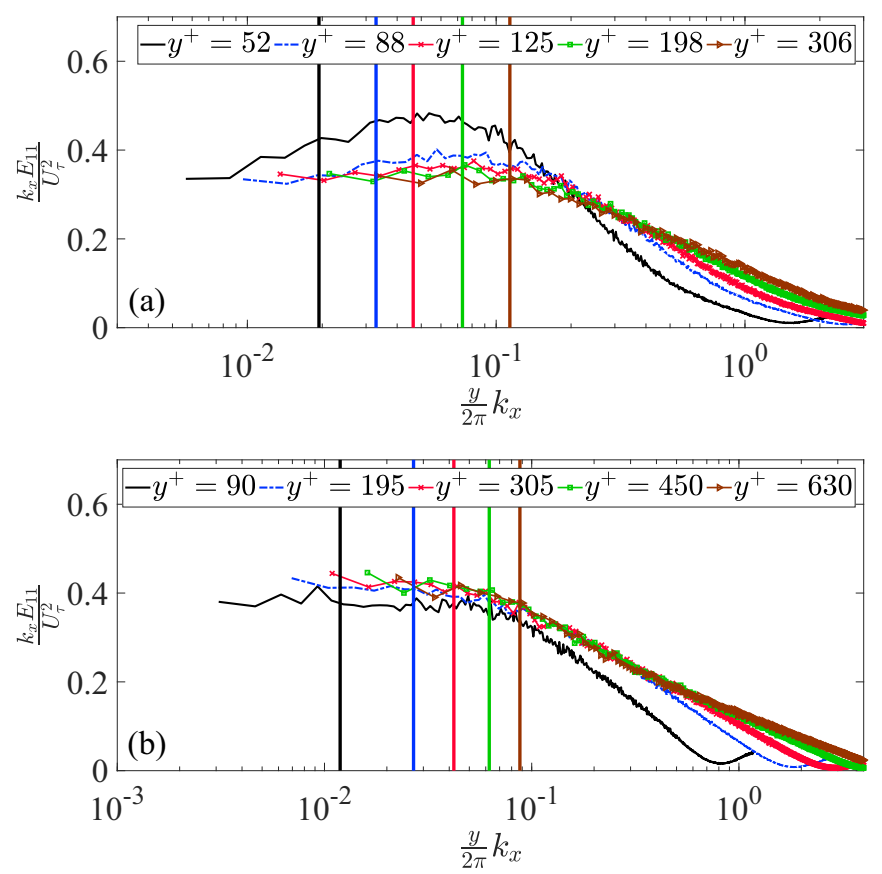

FIG. 8. Same as Fig. 7 in lin-log plots.

A closer look at the spectra in the lin-log plot of Fig. 8 suggests the possibility for small corrections to this conclusion, particularly at the lower of the two $\operatorname{Re}_{\theta}$ values, but the result (10) and (11) of our model in Sec. III may pave the way for a significantly different interpretation. This model leads to $E_{11}\left(k_{x}\right) \sim\left(k_{x} \delta\right)^{q}$ with $p+q=-1$ if $D=1$. Support for $D=$ 1 has been obtained and reported in the previous subsection in the range of lengths $\lambda$ between about $0.5 \delta$ and $2 \delta$. It is therefore worth taking a closer look at our energy spectra in the corresponding wave-number range. For our data, this wave-number range turns out, in fact, to be comparable to the wave-number range $2 \pi /(4 \delta) \lesssim k_{x} \lesssim 0.63 / y$ mentioned in the previous paragraph as a candidate for Townsend-Perry scaling. Specifically, $k_{x} /(2 \pi)=2 / \delta$ corresponds to $k_{x} y /(2 \pi)=0.25$, $0.41,0.58,0.91$, and 1.41 in increasing order of the $y^{+}$values in Figs. 7 and 8 for $\operatorname{Re}_{\theta}=8100$, and to $k_{x} y /(2 \pi)=0.15$, $0.33,0.53,0.78$, and 1.1 in increasing order of the $y^{+}$values in Figs. 7 and 8 for $\operatorname{Re}_{\theta}=20600$. The wave-number range $0.5 / \delta \leqslant k_{x} /(2 \pi) \leqslant 2 / \delta$ where the analysis in the remainder of our paper is carried out is therefore not radically different for our data from the wave-number range $2 \pi /(4 \delta)<k_{x}<0.63 / y$ where one would interpret our spectra to have a TownsendPerry scaling for $y^{+} \geqslant 88$.

In Figs. $9-12$ we plot $\overline{a^{2}}$ versus $\lambda / \delta$ where $\overline{a^{2}}$ is the average of $\alpha^{2} / \Delta \lambda$ conditional on the streamwise length of a labeled structure being between $\lambda$ and $\lambda+\Delta \lambda$ ( $\alpha$ and $\lambda$ being obtained as explained in the first paragraph of Sec. VC). The upper values of $\lambda / \delta$ in these plots are all below about 2.3 because we do not have enough samples of educed structures beyond $\lambda / \delta \approx 2.3$ to obtain values of $\overline{a^{2}}$ which are statistically converged. The lower values of $\lambda / \delta$ in these plots are all close to $1 / 2$ because the range where the PDF of $\lambda / \delta$ has been found in the previous subsection to be well approximated by $-C_{1}+C_{2}(\lambda / \delta)^{-2}$ is bounded from below by about $1 / 2$ in all
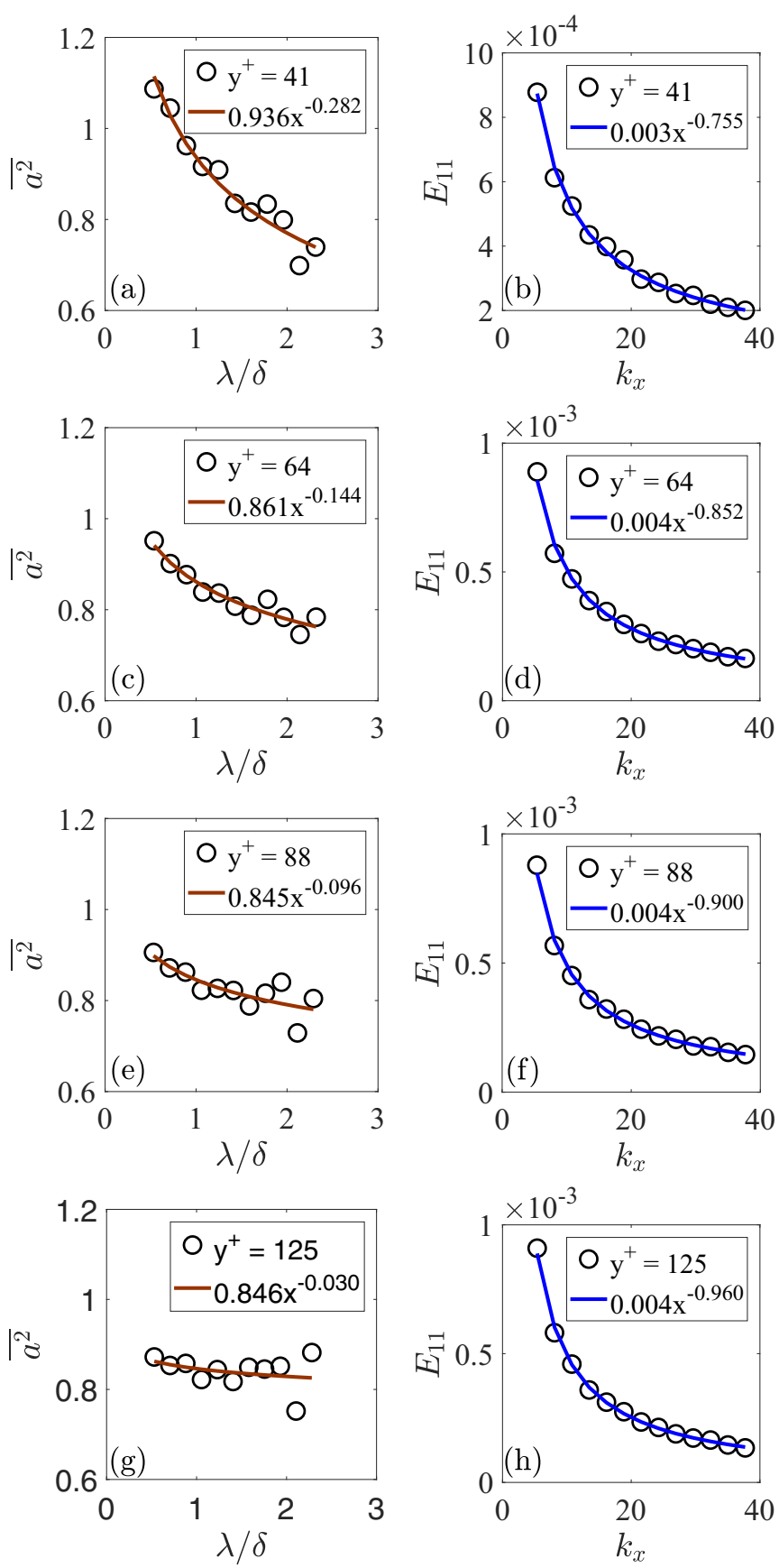

FIG. 9. Lin-lin plots of $\overline{a^{2}}$ versus $\lambda / \delta$ (left) and streamwise energy spectra plotted at wall distances $y^{+}=41,64,88$, and 125 (from top to bottom) at $\operatorname{Re}_{\theta}=8100$.

our $y^{+}$and $\operatorname{Re}_{\theta}$ cases. In Figs. 9-12 we also plot $E_{11}\left(k_{x}\right)$ in the corresponding wave-number range $0.25 / \delta \leqslant k_{x} /(2 \pi) \leqslant 2 / \delta$ which, as discussed in the previous paragraph, may be close to the wave-number range $2 \pi /(4 \delta)<k_{x}<0.63 / y$ that one could interpret as a Townsend-Perry range. We do not have enough data and high enough Reynolds numbers to clearly distinguish between these two ranges in the present work.

As an aside for the moment, note that the large-scale motions (LSMs) and very large-scale motions (VLSMs), which have been found to exist in the logarithmic and lower wake regions of a turbulent boundary layer (see Kovasznay et al. [25], 

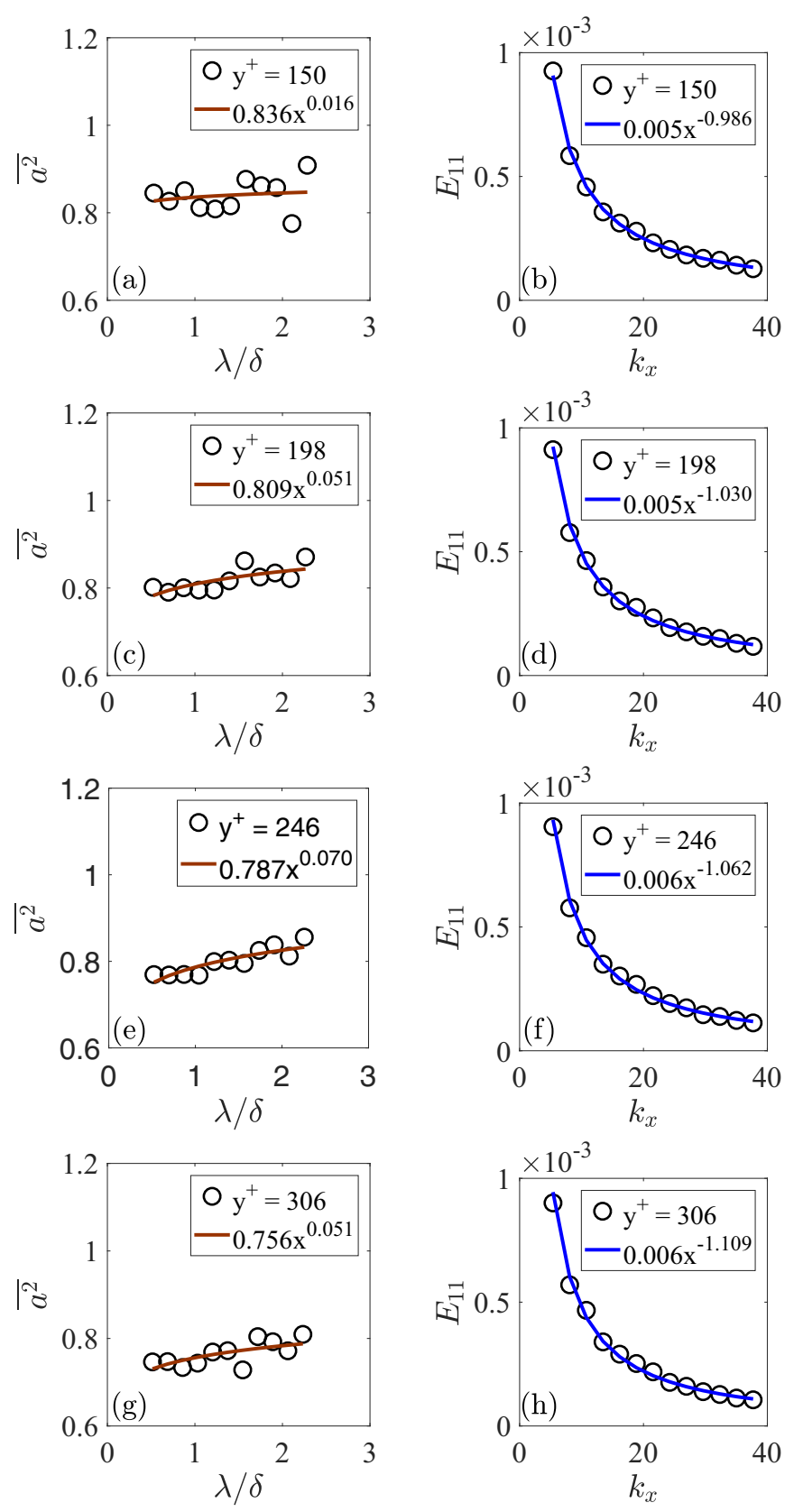

FIG. 10. Lin-lin plots of $\overline{a^{2}}$ versus $\lambda / \delta$ (left) and streamwise energy spectra plotted at wall distances $y^{+}=150,198,246$, and 306 (from top to bottom) at $\operatorname{Re}_{\theta}=8100$.

Brown and Thomas [26], Hutchins and Marusic [27], Dennis and Nickels [28], and Lee and Sung [29]) generally refer to elongated regions of streamwise velocity fluctuations having a streamwise extent from about $2 \delta$ to $3 \delta$ for LSMs and larger than $3 \delta$ for VLSMs (see Kim and Adrian [30], Guala et al. [31], and Balakumar and Adrian [32]). The LSMs near the wall and the VLSMs have been interpreted as being responsible for the $k_{x}^{-1}$ scaling range of the turbulence spectrum (Smits et al. [13]). However, the range of scales we concentrate on in Figs. 9-12 extends up to $2.5 \delta$ and may only include some LSMs.

Returning now to Figs. 9-12, we have included best fits of power-law curves in the plots of $\overline{a^{2}}$ versus $\lambda / \delta$ and of $E_{11}$
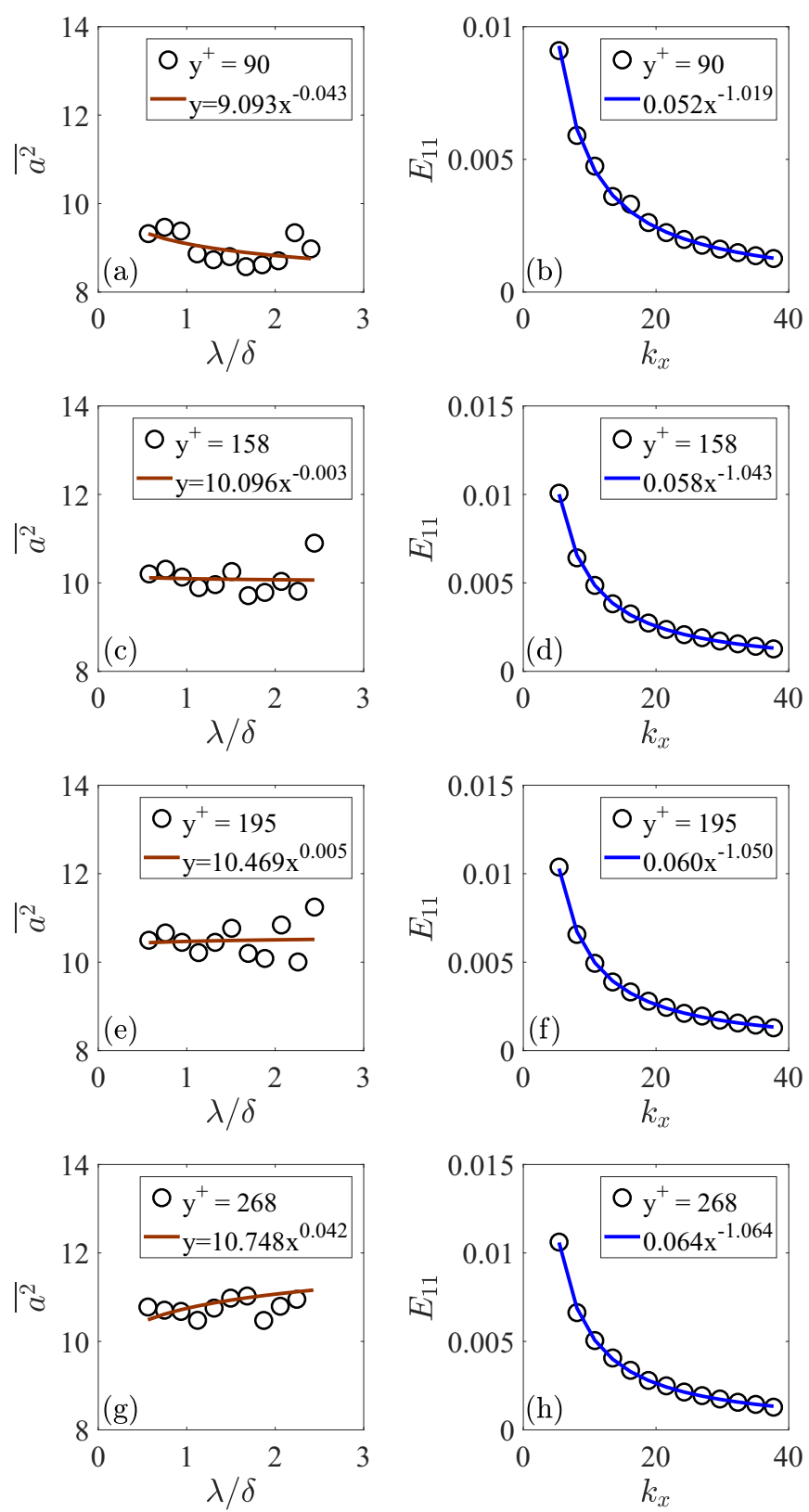

FIG. 11. Lin-lin plots of $\overline{a^{2}}$ versus $\lambda / \delta$ (left) and streamwise energy spectra plotted at wall distances $y^{+}=90,158,195$, and 268 (from top to bottom) at $\mathrm{Re}_{\theta}=20600$.

versus $k_{x}$. These best fits are indicated in the inserts of each plot and provide an estimation of the exponents $p$ and $q$ in $\overline{a^{2}} \sim(\lambda / \delta)^{p}$ and $E_{11}\left(k_{x}\right) \sim k_{x}^{q}$. As the fit of Fig. 9 is computed from $k_{x}=5$ to 40, the PIV transfer function (see Foucaut $e t$ al. [23]) introduces a systematic error on $q$ smaller than $0.5 \%$. Figure 13 summarizes the information with plots of $p, q$, and $p+q$ as functions of $y^{+}$. It is perhaps remarkable that $p+q$ is very close to -1 (see Fig. 13) as predicted by (10) and (11) for all examined values of $y^{+}$and for both Reynolds numbers $\operatorname{Re}_{\theta}$. Whereas this subsection's initial interpretation in terms of the Townsend attached eddy model is limited to $y^{+}$larger or equal to 88 (based on the log-log plots of Fig. 7), the lin-lin plots of Figs. 9-12 present a different and consistent picture 

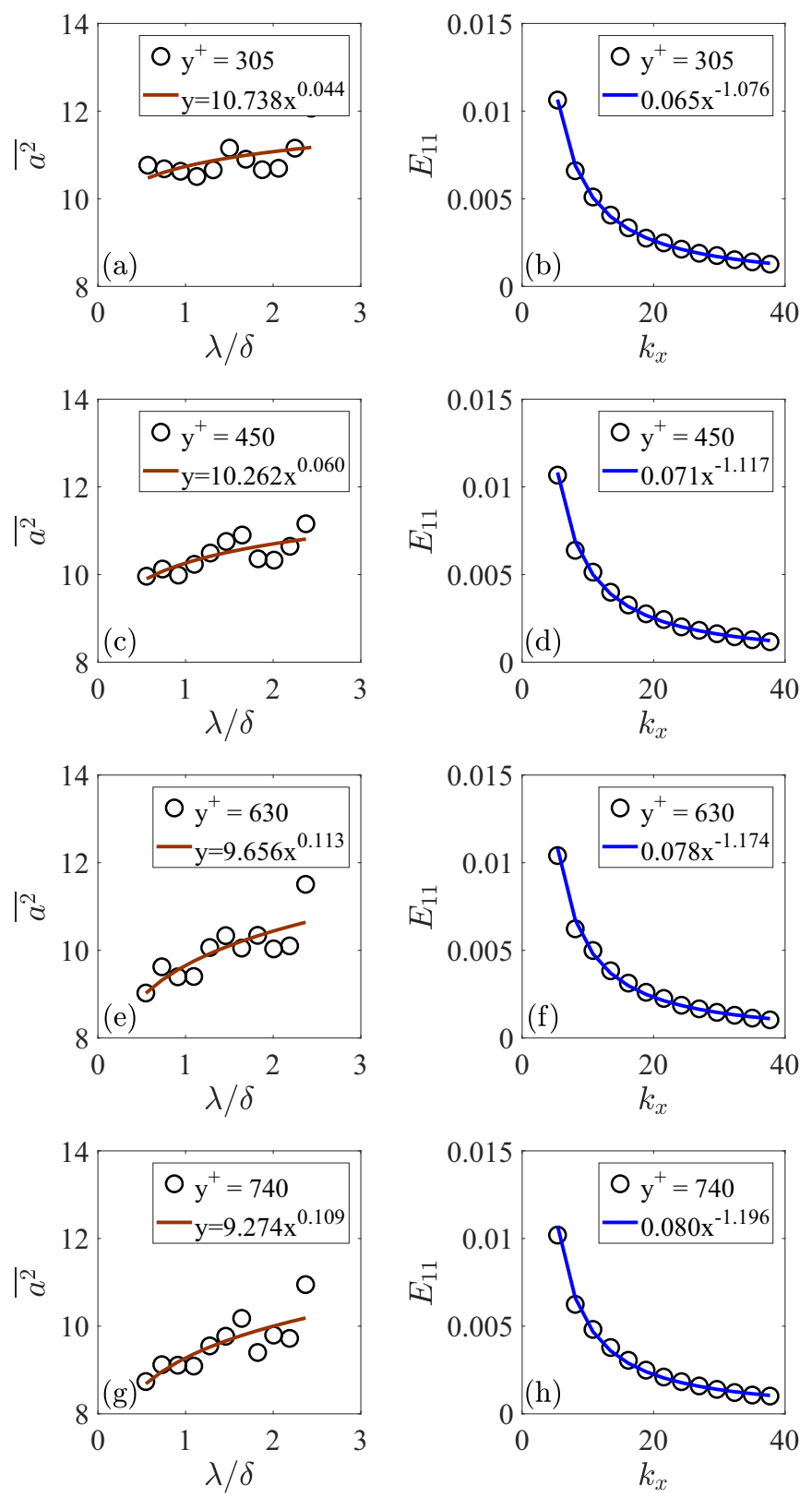

FIG. 12. Lin-lin plots of $\overline{a^{2}}$ versus $\lambda / \delta$ (left) and streamwise energy spectra plotted at wall distances $y^{+}=305,450,630$, and 740 (from top to bottom) at $\operatorname{Re}_{\theta}=20600$.

which covers both Reynolds numbers and all our $y^{+}$positions, including $y^{+}$smaller than 88 . This picture is confirmed by hot-wire anemometry (HWA) data from a turbulent boundary layer in the same wind tunnel by Tutkun et al. [33] and from the recent direct numerical simulations (DNS) of a turbulent channel flow at $\operatorname{Re}_{\tau}=5200$ by Lee and Moser [34]. Indeed, these HWA and DNS data show the same variation of the spectral exponent $q$ with $y^{+}$that we found from using (10) and (11) on our PIV data; see Fig. 14, and also Fig. 13(b) where we collected the values of $q$ from different data. The HWA data, in particular, provide a confirmation of our PIV results because they extend to a wider range at the lower end of wave numbers [see Fig. 14(a) and also Fig. 13(b) where it
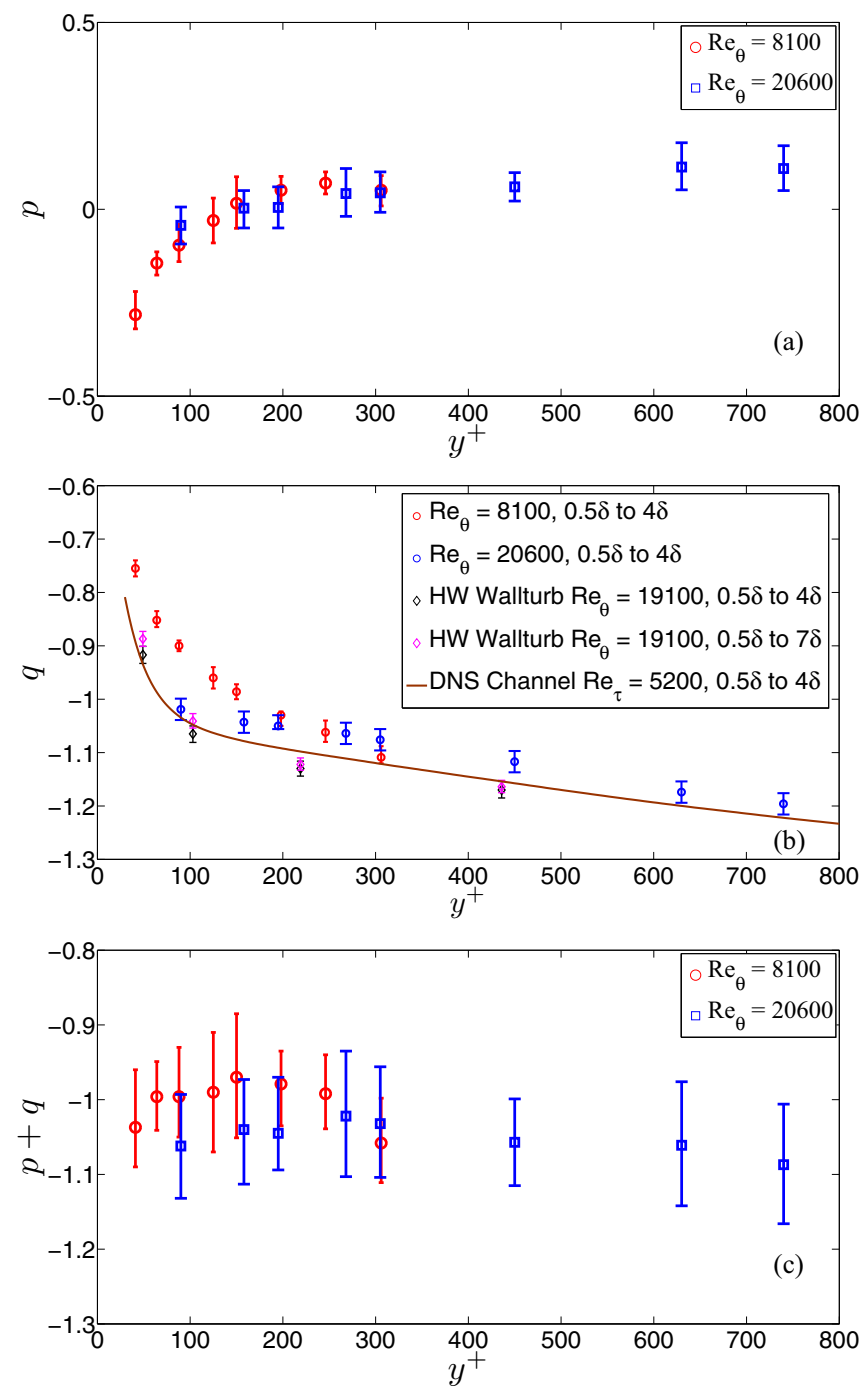

FIG. 13. (a) Exponents $p$ obtained from the best power-law fit of $\overline{a^{2}} \sim(\lambda / \delta)^{p}$. (b) Exponents $q$ obtained from the best power-law fit of $E_{11} \sim k_{x}^{q}$ for the present PIV data, the HWA turbulent boundary layer data of Tutkun et al. [33] \{see Fig. 14(a) and the DNS of turbulent channel flow data of Lee and Moser [34] [see Fig. 14(b)]\}. (c) $p+q$ versus $y^{+}$. All these fits are obtained over the range of scales investigated in Figs. 9-12 [except for the HWA case in (b) where we have also included a fit over a range of length scales extended up to $7 \delta$ ]. The resulting exponents are plotted with the $95 \%$ confidence intervals for these fits.

is shown that the HWA's extended wave-number range returns effectively same values of $q$ ].

The much higher Reynolds number measurements of Vallikivi et al. [12] did not find support for the Townsend-Perry $k_{x}^{-1}$ spectrum either. However, these authors did find some agreement with the $k_{x}^{-1} \log \left(8 \pi / k_{x} y\right)$ spectrum model of del Álamo et al. [35]. This approximate agreement was found in a range of wall-normal distances where we find positive values of $p$, i.e., in a region where the spectrum scales as $k_{x}^{-1} k_{x}^{-p}$ with values of $p$ above but close to 0 . It is quite difficult to distinguish between such a weak power law and $\log \left(8 \pi / k_{x} y\right)$, so the two models qualitatively agree in this range of wall-normal distances. However, the model of del 

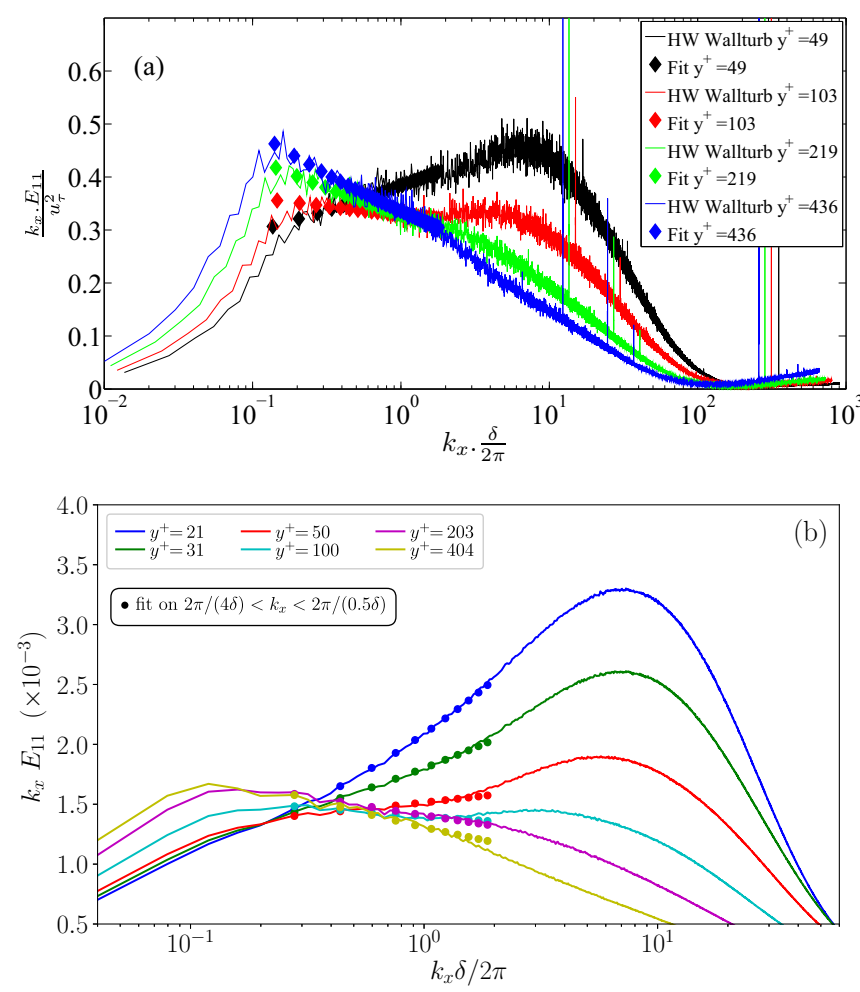

FIG. 14. (a) Lin-log plots of premultiplied streamwise energy spectra at selected wall distances obtained with HWA in the $\operatorname{Re}_{\theta}=$ 19100 turbulent boundary layer of Tutkun et al. [33]. Least-square fits in a range bounded from above by $k_{x} \delta /(2 \pi)=2$ but extended to wave numbers as small as $k_{x} \delta /(2 \pi)$ close to $10^{-1}$. (b) Lin-log plots of premultiplied streamwise energy spectra at selected wall distances from the $\operatorname{Re}_{\tau}=5200$ turbulent channel flow DNS of Lee and Moser [34]. Least-square fits in the range $0.25 \leqslant k_{x} /(2 \pi) \leqslant 2$.

Álamo et al. [35] cannot account for the scaling of the energy spectrum at closer distances to the wall where we find $p \leqslant 0$, whereas our model fits the data in this region too.

\section{E. Discussion}

It is important to stress that the support for (10) and (11) in Figs. 9-12 cannot be obtained without the crucial last step of our structure detection algorithm in Sec. VB which discards structures that are not attached to the wall. The structures which do not touch the wall are in fact less elongated and less intense (i.e., smaller $\overline{a^{2}}$ ) on average. We have checked that if we only consider them, we do not find anything close to $p+q=-1$, i.e., (11).

The attached eddy concept introduced by Townsend [1] is therefore important for explaining $E_{11}\left(k_{x}\right)$ but the results of our analysis suggest that the Townsend-Perry model does not hold without some significant corrections because the turbulent kinetic energy content in these wall-attached flow structures does not just scale with $U_{\tau}$. (If it did, $\overline{a^{2}}$ would scale with $U_{\tau}$ and $p$ would be uniformly 0.) At different $y$ inside such a structure, the level of turbulent kinetic energy depends both on $U_{\tau}$ and on the streamwise length of the structure at that height. Furthermore, this dependence varies with height: $\overline{a^{2}}$ decreases with increasing $\lambda / \delta$ very close to the wall, in the buffer layer, and increases with increasing $\lambda$ further up. As $\overline{a^{2}}$ transits smoothly from one dependence to the other, a particular height exists where $\overline{a^{2}}$ is independent of $\lambda$ and therefore depends only on $U_{\tau}$. At that very particular height, $E_{11}\left(k_{x}\right) \sim k_{x}^{-1}$. However, strictly speaking, this is not a Townsend-Perry spectrum, it is just the spectrum at that particular distance from the wall where the turbulent kinetic energy inside the streaky structures transits from a decreasing to an increasing dependence on the length of these structures. Our conclusion agrees with Nickels et al. [9] in their statement that it is necessary to take measurements close to the wall to observe a $k_{x}^{-1}$ behavior, in fact at $y^{+}$between 100 and 200 as they also found. However, these authors were not in possession of (10) and (11) and therefore did not measure $\overline{a^{2}}$ at various heights and for various values of $\lambda / \delta$ which now allows us to see that the $k_{x}^{-1}$ behavior at the edge of the buffer layer is not the Townsend-Perry spectrum but just a transitional instance of a more involved spectral structure. In fact, the spectral picture which emerges from our analysis is a unified picture which brings together the buffer and inertial layers in a seemless way.

In Fig. 15 we plot examples of measured streamwise velocity fluctuations and the on-off signals with which we model them at various heights from the wall. Our model on-off signals are clearly a drastic simplification of the data but one gets the impression from these plots that they capture the sharpest gradients in the signal and therefore much of its spectral content at the length scales considered here. The lengths of the nonzero parts of the model signals correspond to $\lambda$ and the actual values of the on-off signal in these nonzero parts correspond to the average value $\alpha$ of the streamwise fluctuating velocity component inside each part. We stress that it is enough that our on-off model agrees with the data in the way it does in Figs. 9-12 for a certain range of thresholds $u_{\text {th }}$ (see Sec. V B). Our model does not need to work for any arbitrary threshold; it only needs to work for those thresholds which effectively capture the spatial boundaries of the flow structure objects simulated by our on-off functions as mentioned at the end of the first paragraph of Sec. II.

It is clear that a wider range of Reynolds numbers needs to be examined to establish the scalings of the lower and upper bounds of the range of wave numbers where (10) and (11) hold. One might expect the upper bound to scale as $1 / y$ because of the recent evidence (see Hultmark et al. [36] and Laval et al. [37]) that a Townsend-like approximately logarithmic (or very weak power law) dependence on $y$ exists for the rms streamwise turbulent velocity in the outer part of the inertial range of wall distances. If one assumes the lower bound to scale as $1 / \delta$ and therefore an energy spectrum $E_{11}\left(k_{x}\right)$ of the form (i) $E_{11}\left(k_{x}\right) \sim U_{\tau}^{2} \delta$ for $0 \leqslant k_{x} \leqslant B_{1} / \delta$, (ii) $E_{11}\left(k_{x}\right) \sim U_{\tau}^{2} \delta\left(k_{x} \delta\right)^{-1-p}$ for $B_{1} / \delta \leqslant k_{x} \leqslant B_{2} / y$ (where $B_{1}$ and $B_{2}$ are dimensionless constants and $p$ may be a function of $y$ as in Fig. 13(a), and (iii) comparatively negligible energy at wave numbers $k_{x}>B_{2} / y$, then we should have

$$
\left(u^{\prime+}\right)^{2} \sim 1+\frac{B_{1}^{p}}{p}\left(B_{1}^{-p}-\left(B_{2} \delta / y\right)^{-p}\right) .
$$

This expression for $\left(u^{\prime+}\right)^{2}$ tends to

$$
\left(u^{\prime+}\right)^{2} \sim 1+\left(\ln B_{1}-\ln \left(B_{2} \delta / y\right)\right)
$$



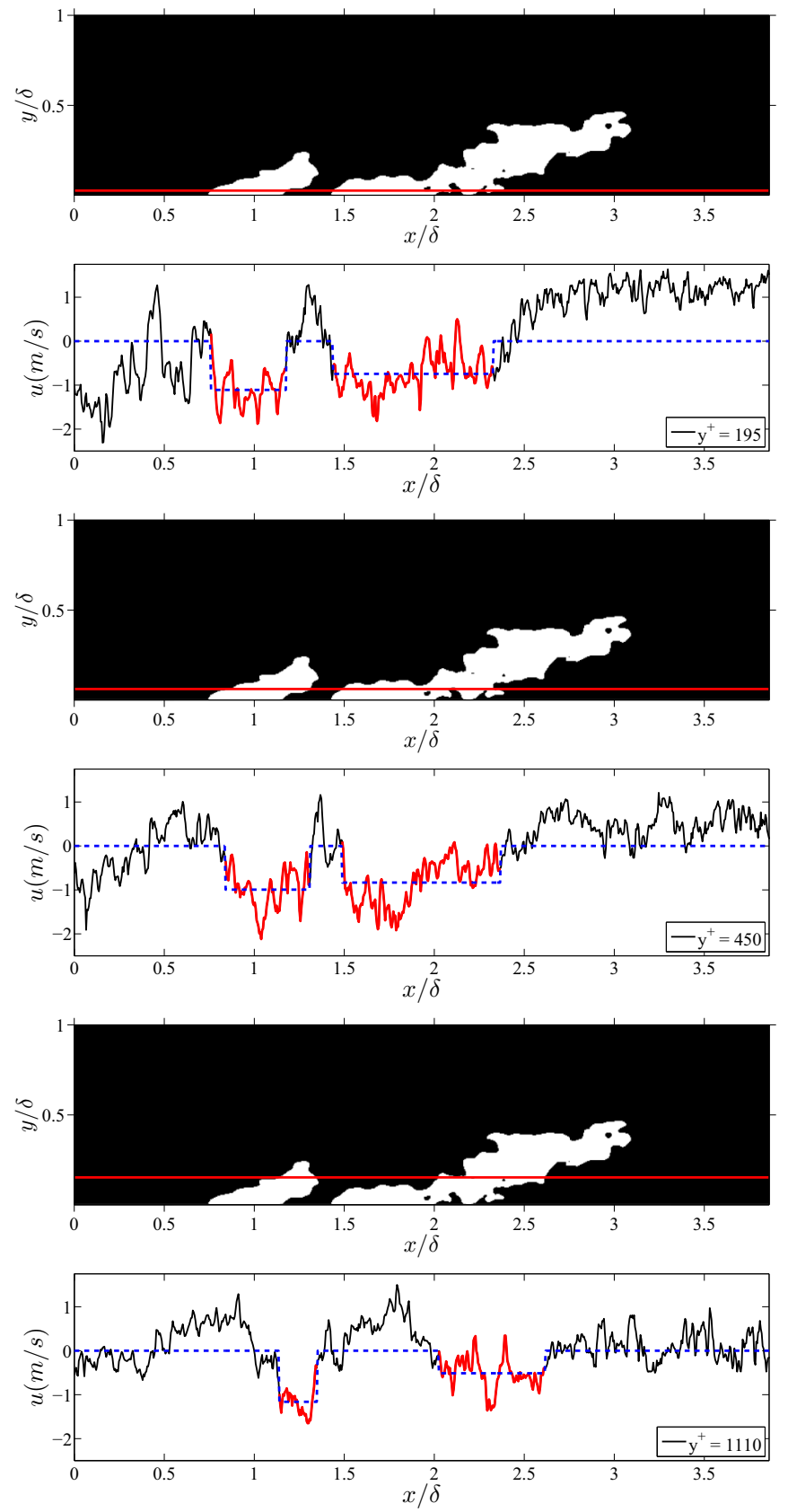

FIG. 15. An example of a detected wall-attached flow structure for $\operatorname{Re}_{\theta}=20600$ and the $u(x)$ signal through this structure at three different $y^{+}$positions. The red line in the repeated binary image indicates the $y^{+}$position where the signal $u(x)$ is recorded $\left(y^{+}=\right.$ $195,450,1110)$. The black (red) line in the $u(x)$ versus $x / \delta$ plots is the raw (unfiltered) PIV streamwise fluctuating velocity outside (inside) the detected flow structures. The dashed blue line is our model signal, equal to 0 outside and to the average value of $u$ inside the detected structures.

as $p \rightarrow 0$ which is the Townsend logarithmic dependence on $y$ corresponding to $p \equiv 0$ (see Townsend [1], Perry and Abell [7], and Perry et al. [3]). This logarithmic dependence (13) results from the assumption that the upper bound of the range of wave numbers where (10) and (11) may hold with $p \equiv 0$ scales as $1 / y$. Slightly nonzero values of $p$ give slight deviations from this logarithmic dependence, of the form (12). The deviation
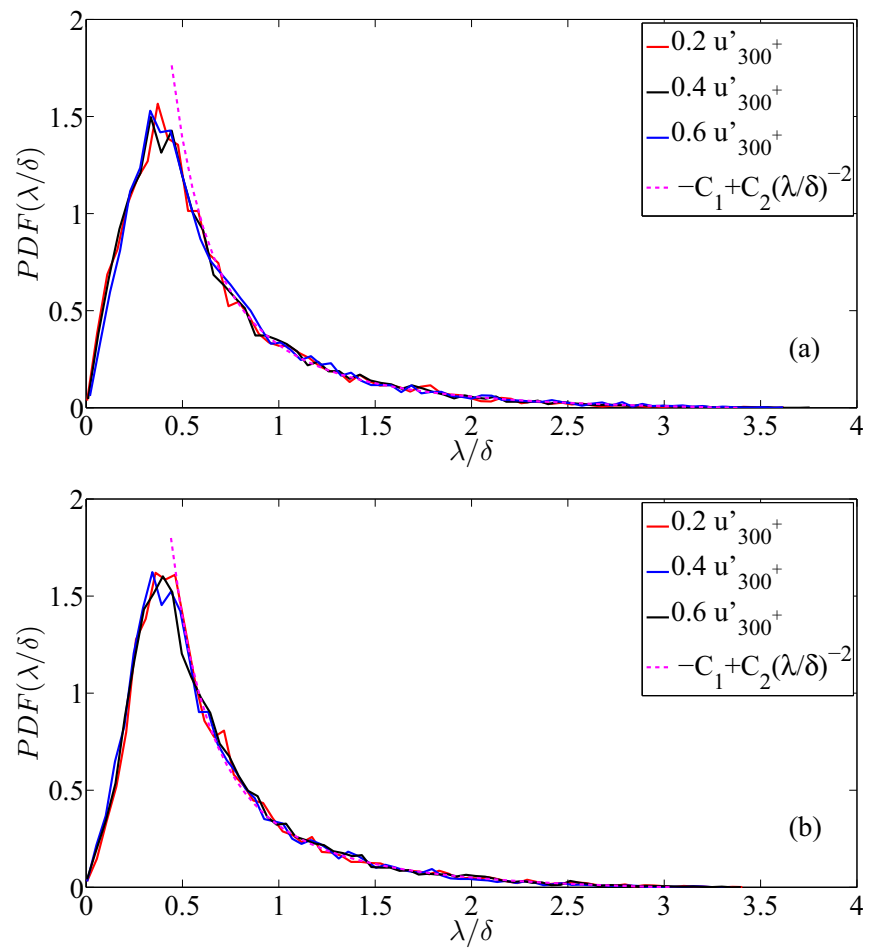

FIG. 16. PDFs of streamwise lengths $\lambda$ of wall-attached structures at $y^{+}=195$ for $\operatorname{Re}_{\theta}=20600$ (top) and $y^{+}=125$ for $\operatorname{Re}_{\theta}=8100$ (bottom) over a set of thresholds.

from Townsend's logarithmic form (13) implied by our work is consistent with the view that the Townsend-Perry constant is not well defined, i.e., not really a constant. This is consistent with the diagnostic plot analysis of Orlu et al. [38].

Using the values of $p$ obtained in this work and plotted versus $y^{+}$in Fig. 13 for our two values of $\operatorname{Re}_{\theta}$, it is not possible to fit (12) to the data in the lower plot of Fig. 1 from $y^{+}=41$ to 306 in the $\operatorname{Re}_{\theta}=8100$ case and from $y^{+}=90$ to 742 in the $\operatorname{Re}_{\theta}=$ 20600 case. These are the $y^{+}$ranges where (10) and (11) have been established for our data and they should therefore also be the ranges where (12) holds if the spectral model of the previous paragraph is good enough. However, in spite of the three adjustable dimensionless constants $\left(B_{1}, B_{2}\right.$, and an overall constant of proportionality), Eq. (12) cannot fit the entire $y^{+}$ range for which this model has been designed, that is, a $y^{+}$ range which includes both the $p<0$ and the $p>0$ regions.

A most suspect part of the spectral model used to derive (12) is its low wave-number part. Vassilicos et al. [39] showed that the second peak or plateau part of the $u^{\prime+}$ profile can be reproduced by a spectral range between the very low wavenumber range where $E_{11}\left(k_{x}\right) \sim U_{\tau}^{2} \delta$ and the wave-number range where $E_{11}\left(k_{x}\right) \sim U_{\tau}^{2} \delta\left(k_{x} \delta\right)^{-1-p}$. In fact, Vassilicos et al. [39] also showed that this extra intermediate spectrum is necessary for a sufficiently fast growth of the integral scale with distance from the wall. A complete model of $E_{11}\left(k_{x}\right)$ would therefore require the spectral range introduced by Vassilicos et al. [39] as well as the spectral range studied here.

\section{CONCLUSION}

We obtained well-resolved PIV data of a flat plate turbulent boundary layer in a large field of view at two Reynolds 

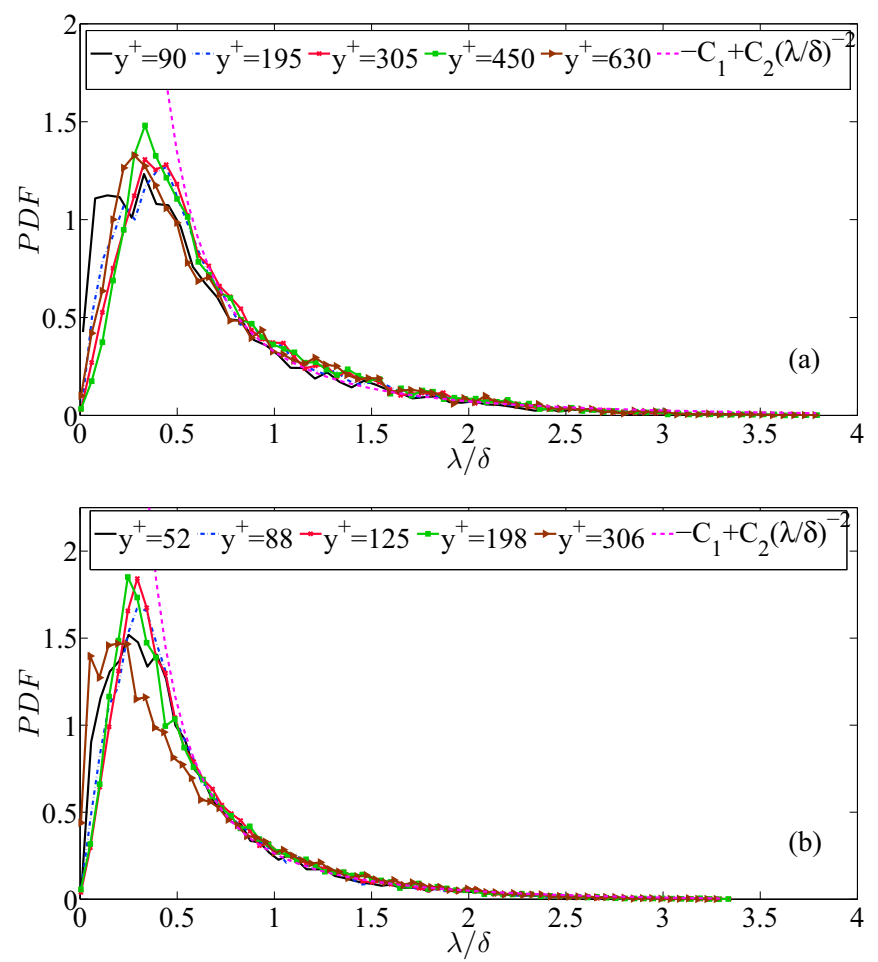

FIG. 17. PDFs of streamwise lengths $\lambda$ of wall-attached structures of positive streamwise fluctuating velocity with $u_{\mathrm{th}}=u_{300^{+}}^{\prime}$ at selected wall distances for $\operatorname{Re}_{\theta}=20600$ (top) and $\operatorname{Re}_{\theta}=8100$ (bottom). The fits shown here are for $y^{+}=195$ at $\operatorname{Re}_{\theta}=20600$ and $y^{+}=198$ at $\operatorname{Re}_{\theta}=8100$.

numbers, $\operatorname{Re}_{\theta}=8100$ and $\operatorname{Re}_{\theta}=20600$. A direct inspection of log-log plots of the streamwise energy spectrum would suggest $E_{11}\left(k_{x}\right) \sim U_{\tau}^{2} k_{x}^{-1}$ in the range $2 \pi /(4 \delta)<k_{x}<0.63 / y$. However, a closer look assisted by relations (10) and (11) reveals a significantly subtler behavior. These relations introduce a specific data analysis which involves the extraction of wall-attached elongated streaky structures from PIV data. The concurrent analysis of streamwise energy spectra and of the relation between the turbulence levels inside streaky structures and the length of these structures offers strong support for (10) and (11) over a significant range of wave numbers and length scales. This range covers LSMs and is comparable to the range where one might have expected the Townsend-Perry attached eddy model spectra to be present. Even though $k_{x}^{-1}$ spectra are not, strictly speaking, validated by our data, the streaky structures which account for the scalings of $E_{11}\left(k_{x}\right)$ do need to be wall attached for relations (10) and (11) to hold. Our conclusions agree with the experiments of Vallikivi et al. [12] which actually suggest that the Townsend-Perry $k_{x}^{-1}$ spectrum cannot be expected even at very high Reynolds numbers. The revised Townsend-Perry streamwise energy spectral form (10) and (11) with $p=p\left(y^{+}\right)$given by Fig. 13(a) appears to extend the validity of the attached eddy concept and its revised consequences to a wider range of Reynolds numbers and a wider range of wall distances.

Finally, we stress that relations (10) and (11) are predicated on these wall-attached streaky structures being space filling,
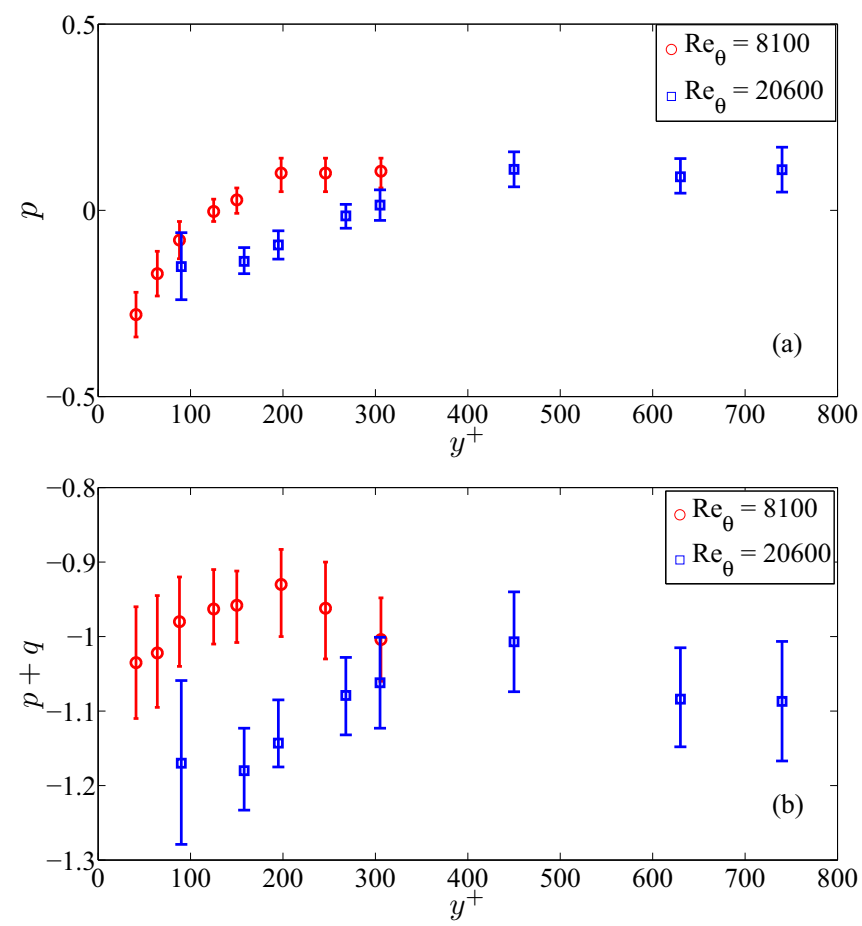

FIG. 18. (a) Exponents $p$ obtained from the best power-law fit of $\overline{a^{2}} \sim(\lambda / \delta)^{p}$. (b) $p+q$ versus $y^{+}$. These fits are obtained over the range of scales investigated for the high-speed regions and the resulting exponents are plotted with the $95 \%$ confidence intervals for these fits. The $y^{+}$positions and the two Reynolds numbers $\operatorname{Re}_{\theta}$ are those in Figs. 9-12.

i.e., $D=1$ in the notation of Sec. II. The pdf of the streamwise length of the educed streaky structures does indeed follow a power law with exponent $-1-D=-2$ over the range of scales which corresponds to the one where (10) and (11) hold.

Our work has shed some new light on the streamwise turbulence spectra of wall turbulence by revealing that some of the inner structure of wall-attached eddies is reflected in the scalings of these spectra via $p\left(y^{+}\right)$. An important implication of this structure is that the friction velocity is not sufficient to scale the spectra. Future work must now further probe the inner structure of wall-attached eddies, attempt to explain it, and extend our analysis to higher Reynolds numbers so as to establish with certainty the ranges of the power laws [exponents $p$ and $q$ in (10) and (11)] discussed in this paper. When this will be done, a complete picture of streamwise energy spectra will also need to integrate the spectral model of Vassilicos et al. [39].

\section{ACKNOWLEDGMENTS}

The work was carried out within the framework of the CNRS Research Foundation on Ground Transport and Mobility, in articulation with the ELSAT2020 project supported by the European Community, the French Ministry of Higher Education and Research, and the Hauts de France Regional Council. The authors gratefully acknowledge the support of these institutions. J.C.V. also acknowledges the support of ERC Advanced Grant No. 320560. 
TABLE II. Number of structures detected over a set of three negative thresholds for $\operatorname{Re}_{\theta}=20600$ and $\operatorname{Re}_{\theta}=8100$.

\begin{tabular}{lcc}
\hline \hline $\operatorname{Re}_{\theta}$ & 20600 & 8100 \\
\hline$-0.2 u_{300^{+}}^{\prime}$ & 13517 & 17338 \\
$-0.4 u_{300^{+}}^{\prime}$ & 14493 & 19576 \\
$-0.6 u_{300^{+}}^{\prime}$ & 13366 & 19290 \\
\hline \hline
\end{tabular}

\section{APPENDIX: EFFECTS OF THRESHOLD LEVELS AND SIGN}

Our results have no significant dependence on threshold $u_{\text {th }}$ in the range from $-0.2 u_{300^{+}}^{\prime}$ to $-0.6 u_{300^{+}}^{\prime}$. An example of this lack of threshold dependence can be seen in the PDFs of $\lambda / \delta$ which we plot in Fig. 16. We also report in Table II the number of structures educed by the algorithm described in Sec. V B for the three negative threshold values $-0.2 u_{300^{+}}^{\prime}$, $-0.4 u_{300^{+}}^{\prime}$, and $-0.6 u_{300^{+}}^{\prime}$. Figures $9-13$ have been obtained for $u_{\text {th }}=-0.4 u_{300^{+}}^{\prime}$ but we checked that they remain very similar without deviations from our conclusions if the threshold $u_{\mathrm{th}}$ is chosen in the range from $-0.2 u_{300^{+}}^{\prime}$ to $-0.6 u_{300^{+}}^{\prime}$.

As mentioned in Sec. VB, this paper's analysis can be repeated equally well on structures of positive streamwise
TABLE III. Values of the constants $C_{1}$ and $C_{2}$ in the form $-C_{1}+$ $C_{2}(\lambda / \delta)^{-2}$ of the PDF of $\lambda / \delta$.

\begin{tabular}{ccccccccccc}
\hline \hline $\operatorname{Re}_{\theta}$ & \multicolumn{1}{c}{20600} & \multicolumn{1}{c}{8100} \\
\hline$y^{+}$ & 90 & 195 & 305 & 450 & 630 & 52 & 88 & 125 & 198 & 306 \\
$C_{1}$ & 0.02 & 0.02 & 0.01 & 0.02 & 0.02 & 0.02 & 0.02 & 0.02 & 0.03 & 0.02 \\
$C_{2}$ & 0.35 & 0.36 & 0.34 & 0.38 & 0.37 & 0.28 & 0.29 & 0.28 & 0.31 & 0.29 \\
\hline \hline
\end{tabular}

fluctuating velocity. We provide examples of results obtained with $u_{\text {th }}=0.4 u_{300^{+}}^{\prime}$ in Fig. 17 and Table III. There are indeed no significant differences in the results for the low and high speed attached flow regions, except for a lower but consistent value of $C_{1}$ and for a consistently lower value of $C_{2}$ in the lower $\operatorname{Re}_{\theta}$ case. Figures 9-13 can be reproduced for this positive threshold $u_{\text {th }}=0.4 u_{300^{+}}^{\prime}$ and show the exact same trend with $p$ increasing while $q$ is decreasing with increasing $y^{+}$. However, whereas $p$ takes values similar to those for $u_{\mathrm{th}}=-0.4 u_{300^{+}}^{\prime}$ in the lower $\operatorname{Re}_{\theta}$ case, it does not do so in the higher $\operatorname{Re}_{\theta}$ case. As a result $p+q$ is quite close to -1 in the lower $\operatorname{Re}_{\theta}$ case but less so, and in fact closer to -1.1 on average, for the higher $\operatorname{Re}_{\theta}$ (see Fig. 18).
[1] A. A. Townsend, The Structure of Turbulent Shear Flow (Cambridge University Press, Cambridge, 1976).

[2] A. E. Perry and M. S. Chong, On the mechanism of wall turbulence, J. Fluid Mech. 119, 173 (1982).

[3] A. E. Perry, S. Henbest, and M. S. Chong, A theoretical and experimental study of wall turbulence, J. Fluid Mech. 165, 163 (1986).

[4] A. E. Perry and J. D. Li, Experimental support for the attachededdy hypothesis in zero-pressure-gradient turbulent boundary layers, J. Fluid Mech. 218, 405 (1990).

[5] I. Marusic, A. K. M. Uddin, and A. E. Perry, Similarity law for the streamwise turbulence intensity in zero-pressure-gradient turbulent boundary layers, Phys. Fluids 9, 3718 (1997).

[6] I. Marusic and G. J. Kunkel, Streamwise turbulence intensity formulation for flat-plate boundary layers, Phys. Fluids 15, 2461 (2003).

[7] A. E. Perry and C. J. Abell, Asymptotic similarity of turbulence structures in smooth-and rough-walled pipes, J. Fluid Mech. 79, 785 (1977).

[8] T. B. Nickels, I. Marusic, S. Hafez, and M. S. Chong, Evidence of the $k_{1}^{-1}$ Law in a High-Reynolds-Number Turbulent Boundary Layer, Phys. Rev. Lett. 95, 074501 (2005).

[9] T. B. Nickels, I. Marusic, S. Hafez, N. Hutchins, and M. S. Chong, Some predictions of the attached eddy model for a high Reynolds number boundary layer, Philos. Trans. R. Soc. Lond. 365, 807 (2007).

[10] J. F. Morrison, B. J. McKeon, W. Jiang, and A. J. Smits, Scaling of the streamwise velocity component in turbulent pipe flow, J. Fluid Mech. 508, 99 (2004).

[11] B. J. McKeon and J. F. Morrison, Asymptotic scaling in turbulent pipe flow, Philos. Trans. R. Soc. A 365, 771 (2007).

[12] M. Vallikivi, B. Ganapathisubramani, and A. J. Smits, Spectral scaling in boundary layers and pipes at very high Reynolds numbers, J. Fluid Mech. 771, 303 (2015).
[13] A. J. Smits, B. J. McKeon, and I. Marusic, High-Reynolds number wall turbulence, Annu. Rev. Fluid Mech. 43, 353 (2011).

[14] J. C. Vassilicos and J. C. R. Hunt, Fractal dimensions and spectra of interfaces with application to turbulence, Proc. R. Soc. Lond. A. 435, 505 (1991).

[15] J. Kostas, J.-M. Foucaut, and M. Stanislas, Application of double SPIV on the near wall turbulence structure of an adverse pressure gradient turbulent boundary layer, in Proceedings of the 6th International Symposium on Particle Image Velocimetry, Pasadena, CA (2005), pp. 21-41.

[16] M. Raffel, C. E. Willert, and J. Kompenhans, Particle Image Velocimetry: A Practical Guide (Springer Science \& Business Media, Medford, 2007).

[17] C. E. Willert and M. Gharib, Digital particle image velocimetry, Exp. Fluids 10, 181 (1991).

[18] J. Soria, An investigation of the near wake of a circular cylinder using a video-based digital cross-correlation particle image velocimetry technique, Exp. Therm. Fluid Sci. 12, 221 (1996).

[19] F. Scarano, Iterative image deformation methods in PIV, Meas. Sci. Technol. 13, R1 (2001).

[20] B. Lecordier and M. Trinite, Advanced PIV algorithms with image distortion validation and comparison using synthetic images of turbulent flow, in Particle Image Velocimetry: Recent Improvements (Springer-Verlag, Berlin, 2004), pp. 115-132.

[21] J. Westerweel and F. Scarano, Universal outlier detection for PIV data, Exp. Fluids 39, 1096 (2005).

[22] J. Carlier and M. Stanislas, Experimental study of eddy structures in a turbulent boundary layer using particle image velocimetry, J. Fluid Mech. 535, 143 (2005).

[23] J.-M. Foucaut, J. Carlier, and M. Stanislas, PIV optimization for the study of turbulent flow using spectral analysis, Meas. Sci. Technol. 15, 1046 (2004).

[24] D. J. C. Dennis and T. B. Nickels, Experimental measurement of large-scale three-dimensional structures in a turbulent 
boundary layer. Part 2. Long structures, J. Fluid Mech. 673, 218 (2011).

[25] L. S. G. Kovasznay, V. Kibens, and R. F. Blackwelder, Largescale motion in the intermittent region of a turbulent boundary layer, J. Fluid Mech. 41, 283 (1970).

[26] G. L. Brown and A. S. Thomas, Large structure in a turbulent boundary layer, Phys. Fluids 20, S243 (1977).

[27] N. Hutchins and I. Marusic, Evidence of very long meandering features in the logarithmic region of turbulent boundary layers, J. Fluid Mech. 579, 1 (2007).

[28] D. J. C. Dennis and T. B. Nickels, Experimental measurement of large-scale three-dimensional structures in a turbulent boundary layer. Part 1. Vortex packets, J. Fluid Mech. 673, 180 (2011).

[29] J. H. Lee and H. J. Sung, Very-large-scale motions in a turbulent boundary layer, J. Fluid Mech. 673, 80 (2011).

[30] K. C. Kim and R. J. Adrian, Very large-scale motion in the outer layer, Phys. Fluids 11, 417 (1999).

[31] M. Guala, S. E. Hommema, and R. J. Adrian, Large-scale and very-large-scale motions in turbulent pipe flow, J. Fluid Mech. 554, 521 (2006).

[32] B. J. Balakumar and R. J. Adrian, Large- and very-large-scale motions in channel and boundary-layer flows, Philos. Trans. R. Soc. Lond. 365, 665 (2007).
[33] M. Tutkun, W. K. George, J. Delville, M. Stanislas, P. B. V. Johansson, J.-M. Foucaut, and S. Coudert, Two-point correlations in high Reynolds number flat plate turbulent boundary layers, J. Turbul. 10, 1 (2009).

[34] M. Lee and R. D. Moser, Direct numerical simulation of turbulent channel flow up to $\operatorname{Re}_{\tau}$ 5200, J. Fluid Mech. 774, 395 (2015).

[35] J. C. del Álamo, J. Jiménez, P. Zandonade, and R. D. Moser, Scaling of the energy spectra of turbulent channels, J. Fluid Mech. 500, 135 (2004).

[36] M. Hultmark, M. Vallikivi, S. C. C. Bailey, and A. J. Smits, Turbulent Pipe Flow at Extreme Reynolds Numbers, Phys. Rev. Lett. 108, 094501 (2012).

[37] J.-P. Laval, J. C. Vassilicos, J.-M. Foucaut, and M. Stanislas, Comparison of turbulence profiles in high-Reynolds-number turbulent boundary layers and validation of a predictive model, J. Fluid Mech. 814, R2 (2017).

[38] R. Örlü, A. Segalini, J. Klewicki, and P. H. Alfredsson, Highorder generalisation of the diagnostic scaling for turbulent boundary layers, J. Turbul. 17, 664 (2016).

[39] J. C. Vassilicos, J.-P. Laval, J.-M. Foucaut, and M. Stanislas, The streamwise turbulence intensity in the intermediate layer of turbulent pipe flow, J. Fluid Mech. 774, 324 (2015). 